\title{
Homogenization of a semilinear variational inequality in a thick multi-level junction
}

\author{
Taras A Mel'nyk and lulia A Nakvasiuk
}

\section{"Correspondence:}

melnyk@imath.kiev.ua

Department of Mathematical

Physics, Faculty of Mathematics and

Mechanics, Taras Shevchenko

National University of Kyiv,

Volodymyrska str. 64, Kyiv, 01601,

Ukraine

\begin{abstract}
We consider a semilinear variation inequality in a thick multi-level junction $\Omega_{\varepsilon}$, which is the union of a domain $\Omega_{0}$ (the junction's body) and a large number of thin cylinders. The thin cylinders are divided into $m$ classes depending on the geometrical characteristics and the semilinear perturbed boundary conditions of the Signorini type given on their lateral surfaces. In addition, the thin cylinders from each class are $\varepsilon$-periodically alternated along some manifold on the boundary of the junction's body.

The purpose is to study the asymptotic behavior of the solution $u_{\varepsilon}$ of this variation inequality as $\varepsilon \rightarrow 0$, i.e. when the number of the thin cylinders from each class infinitely increases and their thickness tends to zero. The passage to the limit is accompanied by special intensity factors $\left\{\varepsilon^{\alpha_{k}}\right\}_{k=1}^{m}$ in the boundary conditions. We establish two qualitatively different cases in the asymptotic behavior of the solution depending on the value of parameters $\left\{\alpha_{k}\right\}_{k=1}^{m}$. For each case we prove a convergence theorem. As a consequence, we see that $u_{\varepsilon}$ converges (as $\varepsilon \rightarrow 0$ ) to the solution of the corresponding nonstandard homogenized problem and show that the semilinear boundary conditions are transformed in the limiting variational inequalities in the region that is filled up by the thin cylinders from each class.
\end{abstract}

MSC: Primary 35B27; 47J20; secondary 35J85; 74K30

Keywords: homogenization; variational inequality; junction

\section{Introduction and statement of the problem}

It is known that some properties of materials are controlled by their geometrical structure. Therefore, the study of the influence of the material microstructure can improve its useful properties and reduce undesirable effects. Mathematical models for this study are boundary-value problems (BVPs) in domains with complex structures: perforated domains, grid-domains, domains with rapidly oscillating boundaries, junctions of thin domains of different configuration, thick junctions, etc.

The present paper is devoted to further development of the asymptotic method proposed in [1], where we studied the linear Signorini boundary-value problem in a thick junction.

A thick junction of type $k: p: d$ is a union of some domain in $\mathbb{R}^{n}$ (called the junction's $b o d y)$ and a large number of thin domains, which are $\varepsilon$-periodically attached to some man-

(c) 2016 Mel'nyk and Nakvasiuk. This article is distributed under the terms of the Creative Commons Attribution 4.0 International License (http://creativecommons.org/licenses/by/4.0/), which permits unrestricted use, distribution, and reproduction in any medium, provided you give appropriate credit to the original author(s) and the source, provide a link to the Creative Commons license, and indicate if changes were made. 
ifold on the boundary of the junction's body. This manifold is called the joint zone. The small parameter $\varepsilon$ characterizes the distance between neighboring thin domains and also their thickness. The type $k: p: d$ of a thick junction refers, respectively, to the limiting dimensions (as $\varepsilon \rightarrow 0$ ) of the junction's body, the joint zone and each of the attached thin domains.

Various constructions of thick-junction type are successfully used in nanotechnologies [2, 3], microtechnique [4], modern engineering constructions (microstrip radiator, ferritefilled rod radiator), as well as many other physical and biological systems such as, for example, efficient sensors (inertial, biological, chemical) (see the review [5]), signal processing filters (ultra large band), micro-fractal constructions, the structure of the intestine lining with different levels of absorption of nutrients on different part of the tissues, and so on.

It is often impossible to solve problems in thick junctions directly with numerical methods, because this would require too much CPU resources considering a large number of components of thick junctions (in some cases few thousands). Therefore development of new mathematical tools is necessary. One of them is asymptotic analysis of BVPs in thick junctions as $\varepsilon \rightarrow 0$, i.e., when the number of attached thin domains infinitely increases and their thickness decreases to zero. Asymptotic results give us the possibility to replace the original problem in a thick junction by the corresponding homogenized problem that is simpler and then apply computer simulation.

\subsection{A model thick multi-level junction}

In contrast to the paper [1] we consider thick junctions with more complex structure, namely thick multi-level junctions.

Let $B$ be a finite union of smooth, disjoint, and nontangent $2 D$-domains $B_{1}, \ldots, B_{m}$ strictly lying in the unit square $\square:=\left\{\xi^{\prime}=\left(\xi_{1}, \xi_{2}\right): 0<\xi_{1}<1,0<\xi_{2}<1\right\}$.

A model thick multi-level junction $\Omega_{\varepsilon}$ of type $3: 2: 1$ consists of the junction body

$$
\Omega_{0}=\left\{x \in \mathbb{R}^{3}: x^{\prime}:=\left(x_{1}, x_{2}\right) \in Q:=(0, a) \times(0, a), 0<x_{3}<\gamma\left(x^{\prime}\right)\right\},
$$

where $\gamma \in C^{1}(\bar{Q})$ and $\min _{x^{\prime} \in \bar{Q}} \gamma\left(x^{\prime}\right)=\gamma_{0}>0$, and a large number of thin cylinders $G_{\varepsilon}:=$ $\bigcup_{k=1}^{m} G_{\varepsilon}(k)$, which are divided into $m$ classes:

$$
G_{\varepsilon}(k)=\bigcup_{i, j=0}^{N-1}\left\{x \in \mathbb{R}^{3}:\left(\frac{x_{1}}{\varepsilon}-i, \frac{x_{2}}{\varepsilon}-j\right) \in B_{k}, x_{3} \in\left(-d_{k}, 0\right]\right\},
$$

$k=1,2, \ldots, m$, i.e., $\Omega_{\varepsilon}=\Omega_{0} \cup G_{\varepsilon}$ (see e.g. Figure 1). Here $N$ is a large natural number, $\varepsilon=\frac{a}{N}$ is a small discrete parameter that characterizes the distance between nearby thin cylinders and their thickness.

The second novelty of this paper is the following nonlinear boundary conditions of the Signorini type:

$$
u_{\varepsilon} \leq g_{k}, \quad a_{k} \partial_{\nu} u_{\varepsilon}+\varepsilon^{\alpha_{k}} h_{k}\left(u_{\varepsilon}\right) \leq 0, \quad\left(u_{\varepsilon}-g_{k}\right)\left(a_{k} \partial_{\nu} u_{\varepsilon}+\varepsilon^{\alpha_{k}} h_{k}\left(u_{\varepsilon}\right)\right)=0
$$

on $S_{\varepsilon}(k), k=1, \ldots, m$, where $\partial_{\nu}=\frac{\partial}{\partial \nu}$ is the outward normal derivative, and $S_{\varepsilon}(k)$ is the union of the lateral surfaces of the thin cylinders $G_{\varepsilon}(k)$. 
Figure 1 Thick two-level junction.

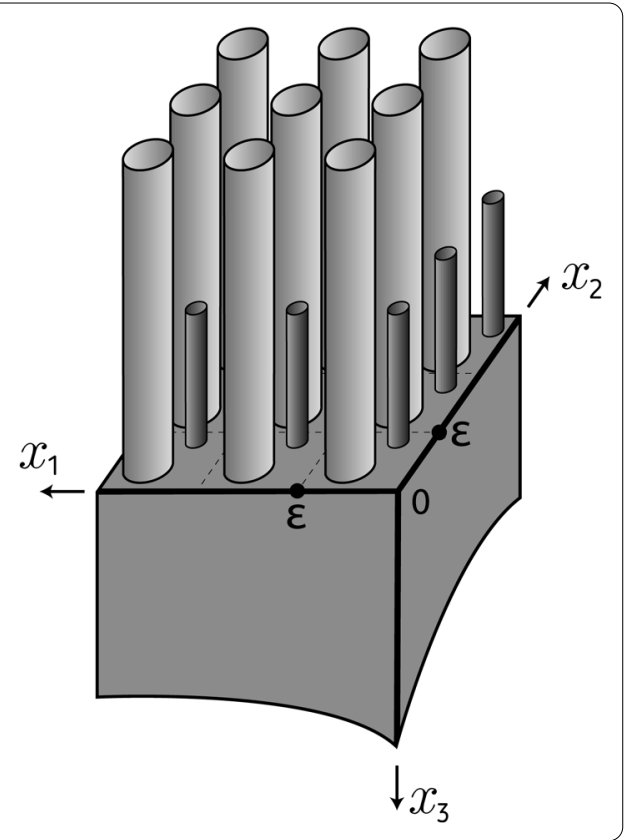

Thus, each class $G_{\varepsilon}(k)$ is characterized by their geometrical characteristics (the crosssectional area $B_{k}$ and the length $d_{k}$ ) and their physical properties described by the positive coefficient $a_{k}$, the functions $\left\{g_{k}, h_{k}, \mu_{k}\right\}$ and the parameter $\alpha_{k} \in \mathbb{R}$.

Many problems in applied mathematics involve the Signorini boundary conditions. Applications arise in groundwater hydrology, in plasticity, in crack theory, in optimal control problems, etc. (see [6]). Many of these problems can be recast as variational inequalities $($ see $[6,7])$.

\subsection{Statement of the problem}

In $\Omega_{\varepsilon}$ we consider the following semilinear problem:

$$
\begin{cases}-\Delta u_{\varepsilon}+\mu_{0}\left(u_{\varepsilon}\right)=f, & \text { in } \Omega_{0}, \\ -a_{k} \Delta u_{\varepsilon}+\mu_{k}\left(u_{\varepsilon}\right)=0, & \text { in } G_{\varepsilon}(k), \\ u_{\varepsilon}=0, & \text { on } \Gamma_{0}, \\ \partial_{\nu} u_{\varepsilon}=0, & \text { on } \partial \Omega_{\varepsilon} \backslash\left(\Gamma_{0} \cup S_{\varepsilon}\right), \\ {\left.\left[u_{\varepsilon}\right]\right|_{x_{3}=0}=0,\left.\quad \partial_{x_{3}} u_{\varepsilon}\right|_{x_{3}=0+0}=\left.a_{k} \partial_{x_{3}} u_{\varepsilon}\right|_{x_{3}=0-0},} & \text { on } Q_{\varepsilon}(k), k=1, \ldots, m,\end{cases}
$$

with the boundary condition (1.2) on $S_{\varepsilon}(k)$, where the brackets denote the jump of the enclosed quantities, $\Gamma_{0}$ is a surface on $\partial \Omega_{0}$ located in $\left\{x: x_{3}>0\right\}$ and $\left|\Gamma_{0}\right|_{2}>0\left(\left|\Gamma_{0}\right|_{2}\right.$ is the surface Lebesgue measure of $\left.\Gamma_{0}\right), Q_{\varepsilon}(k):=\partial \Omega_{0} \cap \partial G_{\varepsilon}(k)$, and $S_{\varepsilon}:=\bigcup_{k=0}^{m} S_{\varepsilon}(k)$.

For the given functions $f,\left\{g_{k}, \mu_{k}, h_{k}\right\}_{k=1}^{m}$ we assume the following conditions:

C1. $f \in L^{2}\left(\Omega_{0}\right) ; g_{k} \in H^{1}\left(D_{k} ; Q\right)=\left\{\varphi \in H^{1}\left(D_{k}\right):\left.\varphi\right|_{Q}=0\right.$ in sense of the trace $\}$, where $D_{k}:=Q \times\left(-d_{k}, 0\right)$ is parallelepiped that is filled up with the thin cylinders $G_{\varepsilon}(k)$ as $\varepsilon \rightarrow 0$; 
C2. functions $\left\{\mu_{k}\right\}_{k=0}^{m}$ and $\left\{h_{k}\right\}_{k=1}^{m}$ are Lipschitz continuous (it is equivalent that they belong to $\left.W_{\text {loc }}^{1, \infty}(\mathbb{R})\right)$ and there exist positive constants $c_{1}>0$ and $c_{2}>0$ such that

$$
c_{1} \leq \mu_{k}^{\prime}(s) \leq c_{2}, \quad c_{1} \leq h_{k}^{\prime}(s) \leq c_{2} \quad \text { for a.e. } s \in \mathbb{R}
$$

C3. in the case if some $\alpha_{k_{0}}<1$ we suppose that $g_{k_{0}} \equiv 0$ and $h_{k_{0}}(0)=0$ (the condition of zero-absorption).

Our goal is to study the asymptotic behavior of the solution $u_{\varepsilon}$ to the problem (1.2)(1.3) as $\varepsilon \rightarrow 0$, i.e. when the number of thin attached cylinders from each class increases unboundedly, while their thickness tends to zero.

The passage to the limit is accompanied by the perturbed coefficients $\left\{\varepsilon^{\alpha_{k}}\right\}_{k=1}^{m}$ in the boundary conditions (1.2). In the paper we also study the influence of these perturbations on the asymptotic behavior of the solution.

\subsection{Comments to the statement and methods of the study. Description of some results}

1. Thick junctions have special character of the connectedness: there are points in a thick junction, which are at a short distance of order $\mathcal{O}(\varepsilon)$, but the length of all curves, which connect these points in the junction, is of order $\mathcal{O}(1)$. As a result, there are no extension operators that would be bounded uniformly in the corresponding Sobolev spaces (see [8]). At the same time the availability of an uniformly bounded family of extension operators is typical supposition in overwhelming majority of the existing homogenization schemes for problems in perforated domains with the Neumann or Robin boundary conditions (see e.g. $[9,10])$. In addition, thick junctions are non-convex domains with non-smooth boundaries. Therefore, solutions of boundary-value problems in such domains have only minimal $H^{1}$-smoothness, while (see e.g. [10]) the $H^{2}$-smoothness of a solution is necessary to prove the convergence theorem. All these factors create special difficulties in the asymptotic analysis of boundary-value problems in thick junctions.

2. In a typical interpretation the solution to the problem (1.2)-(1.3) denotes the density of some quantity (chemical concentration, temperature, electronic potential) at equilibrium within the thick junction $\Omega_{\varepsilon}$. Usually the source of the quantity is located in the junction's body. Therefore, the right-hand side $f$ is defined in $\Omega_{0}$.

3. Standard assumptions for nonlinear terms of semilinear equations are as follows:

- $|\mu(s)| \leq C(1+|s|)$ for each $s \in \mathbb{R}$ and some constant $C$;

- $\mu(s) s \geq C_{1}|s|^{2}-C_{2}$ for all $s \in \mathbb{R}$ and appropriate constants $C_{1}>0, C_{2} \geq 0$.

However, many physical processes, especially in chemistry and medicine, have a monotonous nature. Therefore, it is naturally to impose special monotonous conditions for nonlinear terms. In our case we propose simple conditions (1.4) which are easy to verify. For instance, the function

$$
\mu(s)=\frac{\lambda s}{1+\kappa s} \quad(\text { with } \lambda, \kappa>0)
$$

corresponds to the Michaelis-Menten hypothesis in biochemical reactions and to the Langmuir kinetics adsorption models (see $[10,11]$ for more details) and satisfies condition (1.4) if $f \geq 0$. 
From conditions (1.4) it follows (see [8]) that the inequalities

$$
\begin{array}{ll}
c_{1} s^{2}+\mu_{k}(0) s \leq \mu_{k}(s) s \leq c_{2} s^{2}+\mu_{k}(0) s, & \left|\mu_{k}(s)\right| \leq\left|\mu_{k}(0)\right|+c_{3}|s|, \\
c_{1} s^{2}+h_{k}(0) s \leq h_{k}(s) s \leq c_{2} s^{2}+h_{k}(0) s, & \left|h_{k}(s)\right| \leq\left|h_{k}(0)\right|+c_{3}|s|
\end{array}
$$

are satisfied for all $s \in \mathbb{R}$ and $k=1, \ldots, m$.

4. The boundary conditions (1.2) mean that there is a flux of this quantity through some part of the lateral sides of the thin cylinders. It is evident from the results we have presented that these conditions have a substantial influence on the asymptotic behavior of the solution to the problem (1.2)-(1.3). To study the influence of the boundary interactions on the asymptotic behavior of the solution, we introduce special intensity factors $\varepsilon^{\alpha_{k}}, k=1, \ldots, m$.

If some $\alpha_{k} \geq 1$, then the differential equation $-a_{k} \Delta u_{\varepsilon}+\mu_{k}\left(u_{\varepsilon}\right)=0$ in the thin cylinders $G_{\varepsilon}(k)$ and conditions (1.2) are transformed (as $\varepsilon \rightarrow 0$ ) into the following variational relations:

$$
\left\{\begin{array}{l}
u_{0}^{(k)} \leq g_{k}, \quad-a_{k}\left|B_{k}\right| \partial_{x_{3} x_{3}}^{2} u_{0}^{(k)}+\left|B_{k}\right| \mu_{k}\left(u_{0}^{(k)}\right) \leq-\delta_{\alpha_{k}, 1} l_{k} h_{k}\left(u_{0}^{(k)}\right), \\
\left(u_{0}^{(k)}-g_{k}\right)\left(-a_{k}\left|B_{k}\right| \partial_{x_{3} x_{3}}^{2} u_{0}^{(k)}+\left|B_{k}\right| \mu_{k}\left(u_{0}^{(k)}\right)+\delta_{\alpha_{k}, 1} l_{k} h_{k}\left(u_{0}^{(k)}\right)\right)=0
\end{array}\right.
$$

in $D_{k}$, where $\delta_{\alpha_{k}, 1}$ is the Kronecker delta, $\left|B_{k}\right|$ and $l_{k}$ are, respectively, the area and perimeter of the plane domain $B_{k}$.

If $\alpha_{k}<1$, then the extension ${\tilde{u_{\varepsilon}}}^{(k)}$ by zero of the solution $u_{\varepsilon}$ from $G_{\varepsilon}(k)$ into $D_{k}$ converges strongly in $L^{2}\left(D_{k}\right)$ to 0 as $\varepsilon \rightarrow 0$.

Therefore, we consider two qualitatively different cases.

- For all $k \in\{1, \ldots, m\}$, we assume that the parameters $\alpha_{k} \geq 1$. Then the solution $u_{\varepsilon}$ converges in some sense (see Section 3 ) to the multi-valued function $\mathbf{u}_{0}$, which is a solution of the following semilinear variational inequality:

$$
\left\langle\mathcal{A} \mathbf{u}_{0}, \boldsymbol{\varphi}-\mathbf{u}_{0}\right\rangle \geq\left\langle F, \boldsymbol{\varphi}-\mathbf{u}_{0}\right\rangle \quad \forall \boldsymbol{\varphi} \in \mathcal{K}_{0}
$$

- For some $k=k_{0}$ parameter $\alpha_{k_{0}}<1$ and the other parameters $\left\{\alpha_{k}\right\}$ are greater or equal to one. In this case the problem (1.2)-(1.3) splits (as $\varepsilon \rightarrow 0$ ) into $m$ independent problems: one of them is a semilinear boundary-value problem in the junction's body $\Omega_{0}$, the other ones are semilinear variational inequalities in $D_{k}$ for $k \in\{1, \ldots, m\} \backslash\left\{k_{0}\right\}$ (see Section 4).

The rest of this paper is organized as follows. In Section 2 we prove the existence and uniqueness of a weak solution to the problem (1.2)-(1.3) and the corresponding uniform estimate. Then in Section 3, using the method of monotone operators approach developed in [1], we derive the corresponding nonstandard homogenized problem, establish the existence and uniqueness of a weak solution to the homogenized problem in an anisotropic Sobolev space of multi-valued functions, and prove the convergence theorem in the case if all parameters $\alpha_{k} \geq 1, k=1, \ldots, m$. In Section 4 we study the asymptotic behavior of the solution to the problem (1.2)-(1.3) in the second case. All these results are discussed in Section 5, where we also cite additional literature. 


\section{Variational statements for problem (1.2)-(1.3)}

In the Sobolev space $H^{1}\left(\Omega_{\varepsilon} ; \Gamma_{0}\right)=\left\{u \in H^{1}\left(\Omega_{\varepsilon}\right):\left.u\right|_{\Gamma_{0}}=0\right\}$, we define the subset

$$
\mathcal{K}_{\varepsilon}=\left\{\varphi \in H^{1}\left(\Omega_{\varepsilon} ; \Gamma_{0}\right):\left.\varphi\right|_{S_{\varepsilon}(k)} \leq\left. g_{k}\right|_{S_{\varepsilon}(k)} \text { a.e. on } S_{\varepsilon}(k), k=1, \ldots, m\right\},
$$

where $\left.\psi\right|_{S}$ denotes the trace of a Sobolev function $\psi$ on a surface $S$. Obviously, $\mathcal{K}_{\varepsilon}$ is a closed and convex set for every fixed value of $\varepsilon>0$.

Since for each $k \in\{1, \ldots, m\}$ the function $g_{k}$ belongs to $H^{1}\left(D_{k} ; Q\right)$, we can regard $g_{k}=0$ in $\Omega_{0}$. As a result, the function

$$
\mathcal{G}(x):= \begin{cases}0, & x \in \Omega_{0}, \\ g_{k}(x), & x \in G_{\varepsilon}(k), k=1, \ldots, m,\end{cases}
$$

belongs to $H^{1}\left(\Omega_{\varepsilon} ; \Gamma_{0}\right)$ and $\mathcal{G} \in \mathcal{K}_{\varepsilon}$.

Let us suppose that a classical solution of the problem (1.2)-(1.3) exists. Multiplying the equations of the problem (1.2)-(1.3) by the function $u_{\varepsilon}-\mathcal{G}$, integrating by parts in $\Omega_{\varepsilon}$ and taking into account the boundary conditions for $u_{\varepsilon}$, we find

$$
\begin{aligned}
& \int_{\Omega_{0}} \nabla u_{\varepsilon} \cdot \nabla u_{\varepsilon} d x+\sum_{k=1}^{m} a_{k} \int_{G_{\varepsilon}(k)} \nabla u_{\varepsilon} \cdot \nabla\left(u_{\varepsilon}-g_{k}\right) d x+\int_{\Omega_{0}} \mu_{0}\left(u_{\varepsilon}\right) u_{\varepsilon} d x \\
& \quad+\sum_{k=1}^{m} \int_{G_{\varepsilon}(k)} \mu_{k}\left(u_{\varepsilon}\right)\left(u_{\varepsilon}-g_{k}\right) d x+\sum_{k=1}^{m} \varepsilon^{\alpha_{k}} \int_{S_{\varepsilon}(k)} h_{k}\left(u_{\varepsilon}\right)\left(u_{\varepsilon}-g_{k}\right) d \sigma_{x} \\
& =\int_{\Omega_{0}} f u_{\varepsilon} d x .
\end{aligned}
$$

Now we take any function $\varphi \in \mathcal{K}_{\varepsilon}$ and multiply the equations of the problem (1.2)-(1.3) by the function $\varphi-\mathcal{G}$. Similarly as before, we get

$$
\begin{gathered}
\int_{\Omega_{0}} \nabla u_{\varepsilon} \cdot \nabla \varphi d x+\sum_{k=1}^{m} a_{k} \int_{G_{\varepsilon}(k)} \nabla u_{\varepsilon} \cdot \nabla\left(\varphi-g_{k}\right) d x+\int_{\Omega_{0}} \mu_{0}\left(u_{\varepsilon}\right) \varphi d x \\
\quad+\sum_{k=1}^{m} \int_{G_{\varepsilon}(k)} \mu_{k}\left(u_{\varepsilon}\right)\left(\varphi-g_{k}\right) d x+\sum_{k=1}^{m} \varepsilon^{\alpha_{k}} \int_{S_{\varepsilon}(k)} h_{k}\left(u_{\varepsilon}\right)\left(\varphi-g_{k}\right) d \sigma \\
=\int_{\Omega_{0}} f \varphi d x+\sum_{k=1}^{m} \int_{S_{\varepsilon}(k)}\left(\varphi-g_{k}\right)\left(a_{k} \partial_{\nu} u_{\varepsilon}+\varepsilon^{\alpha_{k}} h_{k}\left(u_{\varepsilon}\right)\right) d \sigma_{x} .
\end{gathered}
$$

Since $\varphi \leq g_{k}$ and $a_{k} \partial_{\nu} u_{\varepsilon}+\varepsilon^{\alpha_{k}} h_{k}\left(u_{\varepsilon}\right) \leq 0$ on $S_{\varepsilon}(k)$,

$$
\sum_{k=1}^{m} \int_{S_{\varepsilon}(k)}\left(\varphi-g_{k}\right)\left(a_{k} \partial_{\nu} u_{\varepsilon}+\varepsilon^{\alpha_{k}} h_{k}\left(u_{\varepsilon}\right)\right) d \sigma_{x} \geq 0
$$


Taking into account (2.4), it follows from (2.3) that

$$
\begin{aligned}
& \int_{\Omega_{0}} \nabla u_{\varepsilon} \cdot \nabla \varphi d x+\sum_{k=1}^{m} a_{k} \int_{G_{\varepsilon}(k)} \nabla u_{\varepsilon} \cdot \nabla\left(\varphi-g_{k}\right) d x+\int_{\Omega_{0}} \mu_{0}\left(u_{\varepsilon}\right) \varphi d x \\
& \quad+\sum_{k=1}^{m} \int_{G_{\varepsilon}(k)} \mu_{k}\left(u_{\varepsilon}\right)\left(\varphi-g_{k}\right) d x+\sum_{k=1}^{m} \varepsilon^{\alpha_{k}} \int_{S_{\varepsilon}(k)} h_{k}\left(u_{\varepsilon}\right)\left(\varphi-g_{k}\right) d \sigma_{x} \\
& \quad \geq \int_{\Omega_{0}} f \varphi d x .
\end{aligned}
$$

Definition 2.1 A function $u_{\varepsilon} \in \mathcal{K}_{\varepsilon}$ is called a weak solution to the problem (1.2)-(1.3) if it satisfies equality (2.2) and inequality (2.5) for any function $\varphi \in \mathcal{K}_{\varepsilon}$.

Let us give variational operator statements for the problem (1.2)-(1.3). For this purpose in the space $H^{1}\left(\Omega_{\varepsilon} ; \Gamma_{0}\right)$ along with the ordinary norm $\|u\|_{H^{1}\left(\Omega_{\varepsilon}\right)}=\left(\int_{\Omega_{\varepsilon}}\left(u^{2}+|\nabla u|^{2}\right) d x\right)^{\frac{1}{2}}$, we introduce the new norm $\|\cdot\|_{\varepsilon}$, which is generated by the scalar product

$$
(u, v)_{\varepsilon}=\int_{\Omega_{\varepsilon}} \nabla u \cdot \nabla v d x, \quad u, v \in H^{1}\left(\Omega_{\varepsilon} ; \Gamma_{0}\right) .
$$

Due to the uniform Dirichlet condition on $\Gamma_{0}$, the norms $\|\cdot\|_{\varepsilon}$ and $\|\cdot\|_{H^{1}\left(\Omega_{\varepsilon}\right)}$ are uniformly equivalent, i.e., there exist constants $C_{1}>0$ and $\varepsilon_{0}>0$ such that for all $\varepsilon \in\left(0, \varepsilon_{0}\right)$ and for all $u \in H^{1}\left(\Omega_{\varepsilon} ; \Gamma_{0}\right)$ the following estimates hold:

$$
\|u\|_{\varepsilon} \leq\|u\|_{H^{1}\left(\Omega_{\varepsilon}\right)} \leq C_{1}\|u\|_{\varepsilon}
$$

Denote by $\left(H^{1}\left(\Omega_{\varepsilon} ; \Gamma_{0}\right)\right)^{*}$ the dual space to $H^{1}\left(\Omega_{\varepsilon} ; \Gamma_{0}\right)$ and defined a nonlinear operator $\mathcal{A}_{\varepsilon}: H^{1}\left(\Omega_{\varepsilon} ; \Gamma_{0}\right) \longmapsto\left(H^{1}\left(\Omega_{\varepsilon} ; \Gamma_{0}\right)\right)^{*}$ through the relation

$$
\begin{aligned}
\left\langle\mathcal{A}_{\varepsilon}(u), v\right\rangle_{\varepsilon}= & \int_{\Omega_{0}} \nabla u \cdot \nabla v d x+\sum_{k=1}^{m} a_{k} \int_{G_{\varepsilon}(k)} \nabla u \cdot \nabla v d x \\
& +\int_{\Omega_{0}} \mu_{0}(u) v d x+\sum_{k=1}^{m} \int_{G_{\varepsilon}(k)} \mu_{k}(u) v d x \\
& +\sum_{k=1}^{m} \varepsilon^{\alpha_{k}} \int_{S_{\varepsilon}(k)} h_{k}(u) v d \sigma_{x} \quad \forall u, v \in H^{1}\left(\Omega_{\varepsilon} ; \Gamma_{0}\right),
\end{aligned}
$$

where $\langle\cdot, \cdot\rangle_{\varepsilon}$ is the duality pairing of $\left(H^{1}\left(\Omega_{\varepsilon} ; \Gamma_{0}\right)\right)^{*}$ and $H^{1}\left(\Omega_{\varepsilon} ; \Gamma_{0}\right)$.

Then Definition 2.1 can be re-written as follows.

Definition 2.2 A function $u_{\varepsilon} \in \mathcal{K}_{\varepsilon}$ is called a weak solution to the problem (1.2)-(1.3) if it satisfies the equality

$$
\left\langle\mathcal{A}_{\varepsilon}\left(u_{\varepsilon}\right), u_{\varepsilon}-\mathcal{G}\right\rangle_{\varepsilon}=\left\langle F, u_{\varepsilon}-\mathcal{G}\right\rangle_{\varepsilon}
$$

and inequality

$$
\left\langle\mathcal{A}_{\varepsilon}\left(u_{\varepsilon}\right), \varphi-\mathcal{G}\right\rangle_{\varepsilon} \geq\langle F, \varphi-\mathcal{G}\rangle_{\varepsilon} \quad \forall \varphi \in \mathcal{K}_{\varepsilon} .
$$


In (2.7) and (2.8) $F \in\left(H^{1}\left(\Omega_{\varepsilon} ; \Gamma_{0}\right)\right)^{*}$, it is defined by $\langle F, v\rangle_{\varepsilon}:=\int_{\Omega_{0}} f v d x$. Obviously $\|F\| \leq$ $C\|f\|_{L^{2}\left(\Omega_{0}\right)}$.

An equivalent definition reads as follows.

Definition 2.3 A function $u_{\varepsilon} \in \mathcal{K}_{\varepsilon}$ is called a weak solution to the problem (1.2)-(1.3) if it satisfies the inequality

$$
\left\langle\mathcal{A}_{\varepsilon}\left(u_{\varepsilon}\right), \varphi-u_{\varepsilon}\right\rangle_{\varepsilon} \geq\left\langle F, \varphi-u_{\varepsilon}\right\rangle_{\varepsilon} \quad \forall \varphi \in \mathcal{K}_{\varepsilon}
$$

Let us show that definitions 2.2 and 2.3 indeed are equivalent. Subtracting equality (2.7) from inequality (2.8), we arrive at (2.9). Setting $\varphi=\mathcal{G}$ into (2.9), we have

$$
\left\langle\mathcal{A}_{\varepsilon}\left(u_{\varepsilon}\right), \mathcal{G}-u_{\varepsilon}\right\rangle_{\varepsilon} \geq\left\langle F, \mathcal{G}-u_{\varepsilon}\right\rangle_{\varepsilon}
$$

Putting $\varphi=2 u_{\varepsilon}-\mathcal{G}$ into (2.9), we get the reversed inequality

$$
\left\langle\mathcal{A}_{\varepsilon}\left(u_{\varepsilon}\right), u_{\varepsilon}-\mathcal{G}\right\rangle_{\varepsilon} \geq\left\langle F, u_{\varepsilon}-\mathcal{G}\right\rangle_{\varepsilon}
$$

This means that (2.7) holds. Setting $\varphi=\psi+u_{\varepsilon}-\mathcal{G}$ in (2.9), where $\psi$ is an arbitrary function from $\mathcal{K}_{\varepsilon}$, we get (2.8).

\subsection{Existence and uniqueness of the weak solution. Uniform estimate}

In the following we will often use the following identities (see [8])

$$
\begin{aligned}
\varepsilon \int_{S_{\varepsilon}(k)} v d \sigma_{x}= & \frac{l_{k}}{\left|B_{k}\right|} \int_{G_{\varepsilon}(k)} v d x \\
& +\left.\varepsilon \int_{G_{\varepsilon}(k)} \nabla_{\xi^{\prime}} Y_{k}\left(\xi^{\prime}\right)\right|_{\xi^{\prime}=\frac{x^{\prime}}{\varepsilon}} \cdot \nabla_{x^{\prime}} v d x \quad \forall v \in H^{1}\left(G_{\varepsilon}(k)\right),
\end{aligned}
$$

$k=1, \ldots, m$. Here $\left|B_{k}\right|$ is the area of the plane domain $B_{k}, l_{k}$ is the length of $\partial B_{k}$, the auxiliary function $Y_{k}$ is a unique solution to the following problem:

$$
\begin{cases}\Delta_{\xi} Y_{k}\left(\xi^{\prime}\right)=l_{k}\left|B_{k}\right|^{-1}, & \xi^{\prime}=\left(\xi_{1}, \xi_{2}\right) \in B_{k} \\ \partial_{\nu\left(\xi^{\prime}\right)} Y_{k}\left(\xi^{\prime}\right)=1, & \xi^{\prime} \in \partial B_{k}, \\ \int_{B_{k}} Y_{k}\left(\xi^{\prime}\right) d \xi^{\prime}=0, & \end{cases}
$$

and then $Y_{k}$ is $\varepsilon$-periodically continued with respect to $\xi_{1}$ and $\xi_{2}$. Due to the regularity properties of solutions to elliptic boundary-value problems we have

$$
\sup _{\xi^{\prime} \in B_{k}}\left|\nabla_{\xi^{\prime}} Y_{k}\left(\xi^{\prime}\right)\right| \leq \widetilde{c}_{k}
$$

Using Cauchy's inequality with $\delta\left(a b \leq \delta a^{2}+\frac{b^{2}}{4 \delta}, a, b>0\right)$ and (2.12), we deduce from (2.10) the following estimates:

$$
\varepsilon \int_{S_{\varepsilon}(k)} v^{2} d \sigma_{x} \leq C_{1}\left(\varepsilon^{2} \int_{G_{\varepsilon}(k)}\left|\nabla_{x^{\prime}} v\right|^{2} d x+\int_{G_{\varepsilon}(k)} v^{2} d x\right)
$$




$$
\int_{G_{\varepsilon}(k)} v^{2} d x \leq C_{2}\left(\varepsilon^{2} \int_{G_{\varepsilon}(k)}\left|\nabla_{x^{\prime}} v\right|^{2} d x+\varepsilon \int_{S_{\varepsilon}(k)} v^{2} d \sigma_{x}\right)
$$

for arbitrary function $v \in H^{1}\left(G_{\varepsilon}(k)\right), k=1, \ldots, m$.

Remark 2.1 In (2.13), (2.14), and in the following all constants $C_{i}$ and $c_{i}$ in inequalities are independent of the parameter $\varepsilon$.

To prove the well-posedness result, we verify some properties of the operator $\mathcal{A}_{\varepsilon}$.

1. Using (1.5) and (1.6), and Cauchy's inequality with $\delta>0$, we obtain

$$
\begin{aligned}
& \left\langle\mathcal{A}_{\varepsilon}(v), v\right\rangle_{\varepsilon} \\
& \geq c_{0} \int_{\Omega_{\varepsilon}}|\nabla v|^{2} d x+c_{1} \int_{\Omega_{0}} v^{2} d x+\int_{\Omega_{0}} \mu_{0}(0) v d x+\sum_{k=1}^{m} c_{1} \int_{G_{\varepsilon}(k)} v^{2} d x \\
& \quad+\sum_{k=1}^{m} \int_{G_{\varepsilon}} \mu_{k}(0) v d x+\sum_{k=1}^{m} c_{1} \varepsilon^{\alpha_{k}} \int_{S_{\varepsilon}(k)} v^{2} d \sigma_{x}+\sum_{k=1}^{m} \varepsilon^{\alpha_{k}} \int_{S_{\varepsilon}(k)} h_{k}(0) v d \sigma_{x} \\
& \geq c_{4}\|v\|_{H^{1}\left(\Omega_{\varepsilon}\right)}^{2} \\
& \quad-\delta\left(\left|\mu_{0}(0)\right| \int_{\Omega_{0}} v^{2} d x+\sum_{k=1}^{m}\left|\mu_{k}(0)\right| \int_{G_{\varepsilon}(k)} v^{2} d x+\sum_{k=1}^{m} \varepsilon^{\alpha_{k}}\left|h_{k}(0)\right| \int_{S_{\varepsilon}(k)} v^{2} d \sigma_{x}\right) \\
& \quad-\frac{1}{4 \delta}\left(\left|\mu_{0}(0)\right|\left|\Omega_{0}\right|_{3}+\sum_{k=1}^{m}\left|\mu_{k}(0)\right|\left|G_{\varepsilon}(k)\right|+\sum_{k=1}^{m} \varepsilon^{\alpha_{k}}\left|h_{k}(0)\right|\left|S_{\varepsilon}(k)\right|\right),
\end{aligned}
$$

where $c_{0}=\min \left(1, a_{1}, \ldots, a_{m}\right)$ and $c_{4}=\min \left(c_{0}, c_{1}\right)$. Here it should be noted that the total measure $\left|G_{\varepsilon}(k)\right|$ of the thin cylinders is order of $\mathcal{O}(1)$ and the total measure $\left|S_{\varepsilon}(k)\right|$ of the lateral surfaces of the thin cylinders $G_{\varepsilon}(k)$ is order of $\mathcal{O}\left(\varepsilon^{-1}\right)$.

Then taking into account the condition of zero-absorption if some $\alpha_{k}<1$ and using (2.13), we can select appropriate $\delta$ such that

$$
\left\langle\mathcal{A}_{\varepsilon} v, v\right\rangle_{\varepsilon} \geq C_{1}\|v\|_{\varepsilon}^{2}-C_{2} \quad \forall v \in H^{1}\left(\Omega_{\varepsilon} ; \Gamma_{0}\right) .
$$

This inequality means that the operator $\mathcal{A}_{\varepsilon}$ is coercive.

2. Let us show that it is monotone. Taking into account (1.4), we get

$$
\begin{aligned}
& \left\langle\mathcal{A}_{\varepsilon}\left(u_{1}\right)-\mathcal{A}_{\varepsilon}\left(u_{2}\right), u_{1}-u_{2}\right\rangle_{\varepsilon} \\
& \geq \int_{\Omega_{0}}\left|\nabla\left(u_{1}-u_{2}\right)\right|^{2} d x+\sum_{k=1}^{m} a_{k} \int_{G_{\varepsilon}(k)}\left|\nabla\left(u_{1}-u_{2}\right)\right|^{2} d x+c_{1} \int_{\Omega_{0}}\left(u_{1}-u_{2}\right)^{2} d x \\
& \quad+c_{1} \sum_{k=1}^{m} \int_{G_{\varepsilon}(k)}\left(u_{1}-u_{2}\right)^{2} d x+\sum_{k=1}^{m} \varepsilon^{\alpha_{k}} c_{1} \int_{S_{\varepsilon}(k)}\left(u_{1}-u_{2}\right)^{2} d \sigma \\
& \geq c_{0}\left\|u_{1}-u_{2}\right\|_{\varepsilon}^{2} .
\end{aligned}
$$

3. The operator $\mathcal{A}_{\varepsilon}$ is hemicontinuous. Indeed, the real valued function

$$
[0,1] \ni \tau \rightarrow\left\langle\mathcal{A}_{\varepsilon}\left(u_{1}+\tau v\right), u_{2}\right\rangle_{\varepsilon}
$$


is continuous on $[0,1]$ for all fixed $u_{1}, u_{2}, v \in K_{\varepsilon}$ due to the continuity of the functions $\mu_{0}$, $\mu_{k}, h_{k}$, and Lebesgue's dominated convergence theorem.

4. Let us prove that operator $\mathcal{A}_{\varepsilon}$ is bounded. Using the Cauchy-Bunyakovsky integral inequality, (2.6) and (1.5)-(1.6), we deduce the following inequality:

$$
\begin{aligned}
& \left|\left\langle\mathcal{A}_{\varepsilon}(u), v\right\rangle_{\varepsilon}\right| \\
& \leq c_{5} \int_{\Omega_{\varepsilon}} \nabla u \cdot \nabla v d x+\int_{\Omega_{0}}\left(\left|\mu_{0}(0)\right|+c_{3}|u|\right)|v| d x \\
& \quad+\sum_{k=1}^{m} \int_{G_{\varepsilon}(k)}\left(\left|\mu_{k}(0)\right|+c_{3}|u|\right)|v| d x+\sum_{k=1}^{m} \varepsilon^{\alpha_{k}} \int_{S_{\varepsilon}(k)}\left(\left|h_{k}(0)\right|+c_{3}|u|\right)|v| d \sigma_{x} \\
& \leq c_{5}\|u\|_{\varepsilon}\|v\|_{\varepsilon}+c_{6}\|v\|_{\varepsilon}+\sum_{k=1}^{m} \varepsilon^{\alpha_{k}-1}\left|h_{k}(0)\right| \sqrt{\varepsilon\left|S_{\varepsilon(k)}\right|} \sqrt{\varepsilon \int_{S_{\varepsilon}(k)} v^{2} d \sigma_{x}} \\
& \quad+c_{3} \sum_{k=1}^{m} \varepsilon^{\alpha_{k}-1} \sqrt{\varepsilon \int_{S_{\varepsilon}(k)} u^{2} d \sigma_{x}} \sqrt{\varepsilon \int_{S_{\varepsilon}(k)} v^{2} d \sigma_{x} .}
\end{aligned}
$$

Now, with the help of (2.13) and the condition of zero-absorption in the case if some $\alpha_{k_{0}}<1$, we obtain

$$
\left|\left\langle\mathcal{A}_{\varepsilon}(u), v\right\rangle_{\varepsilon}\right| \leq C_{0}\left(1+\|u\|_{\varepsilon}+\|u\|_{\varepsilon} \sum_{k=1}^{m} \varepsilon^{\alpha_{k}-1}\right)\|v\|_{\varepsilon} \quad \forall u, v \in H^{1}\left(\Omega_{\varepsilon} ; \Gamma_{0}\right) .
$$

Thus, the operator $\mathcal{A}_{\varepsilon}$ is bounded, strongly monotone, and hemicontinuous, i.e., it is pseudo monotone operator (see Proposition 2.5 from [7], Section 2.4). As a result, existence and uniqueness of a solution of the variational inequality (2.9) for every fixed value $\varepsilon$ now follow directly from Theorems 8.2, 8.3 (see [7]).

5. Taking into account (2.15), (2.16) and condition C3, we derive from (2.7) that

$$
C_{1}\left\|u_{\varepsilon}\right\|_{\varepsilon}^{2}-C_{2} \leq 3 \delta\left\|u_{\varepsilon}\right\|_{\varepsilon}^{2}+c_{4}+\frac{c_{5}}{4 \delta}\|\mathcal{G}\|_{\varepsilon}^{2}+\frac{c_{6}}{4 \delta}\|f\|_{L^{2}\left(\Omega_{0}\right)}^{2} .
$$

Selecting appropriate $\delta>0$, we obtain the uniform estimate

$$
\left\|u_{\varepsilon}\right\|_{\varepsilon}^{2} \leq C_{0}\left(1+\|f\|_{L^{2}\left(\Omega_{0}\right)}^{2}+\sum_{k=1}^{m}\left\|g_{k}\right\|_{H^{1}\left(D_{k}\right)}^{2}\right)
$$

3 Convergence theorem in the case $\alpha_{k} \geq 1, k=1, \ldots, m$

For every $k \in\{1, \ldots, m\}$ let us introduce the following extensions by zero:

$$
{\widetilde{v_{\varepsilon}}}^{(k)}(x)= \begin{cases}v_{\varepsilon}, & x \in G_{\varepsilon}(k), \\ 0, & x \in D_{k} \backslash G_{\varepsilon}(k),\end{cases}
$$

where $D_{k}=Q \times\left(-d_{k}, 0\right)$ is parallelepiped that filled up with the thin cylinders $G_{\varepsilon}(k)$ as $\varepsilon \rightarrow 0$. It is obvious that this extension belongs to the anisotropic Sobolev space $W^{0,0,1}\left(D_{k}\right):=\left\{v \in L^{2}\left(D_{k}\right): \exists\right.$ weak derivative $\left.\partial_{x_{3}} v \in L^{2}\left(D_{k}\right)\right\}$ with the scalar product

$$
(u, v)_{W^{0,0,1}\left(D_{k}\right)}=\int_{D_{k}}\left(u v+\partial_{x_{3}} u \partial_{x_{3}} v\right) d x .
$$


Theorem 3.1 (The case $\left.\alpha_{k} \geq 1, k=1, \ldots, m\right)$ The sequence of the weak solutions $\left\{u_{\varepsilon}\right\}_{\varepsilon>0}$ of the problem (1.2)-(1.3) satisfies the following relations:

$$
\left.\begin{array}{ll}
u_{\varepsilon} \mid \Omega_{0} \stackrel{w}{\longrightarrow} u_{0}^{+} & \text {weakly in } H^{1}\left(\Omega_{0} ; \Gamma_{0}\right), \\
\widetilde{u_{\varepsilon}(k)} \stackrel{w}{\longrightarrow}\left|B_{k}\right| u_{0}^{(k)} & \text { weakly in } W^{0,0,1}\left(D_{k}\right), \\
\widetilde{\partial_{x_{i}} u_{\varepsilon}} \stackrel{(k)}{\longrightarrow} 0 & \text { weakly in } L^{2}\left(D_{k}\right), i=1,2,
\end{array}\right\} \text { as } \varepsilon \rightarrow 0
$$

for $k=1, \ldots, m$, and the multi-valued function

$$
\mathbf{u}_{0}(x):= \begin{cases}u_{0}^{+}, & x \in \Omega_{0}, \\ u_{0}^{(k)}, & x \in D_{k}, k=1, \ldots, m\end{cases}
$$

is the unique weak solution to the following problem:

$$
\left\{\begin{array}{l}
-\Delta_{x} u_{0}^{+}+\mu_{0}\left(u_{0}^{+}\right)=f \quad \text { in } \Omega_{0}, \\
u_{0}^{+}=0 \quad \text { on } \Gamma_{0}, \quad \partial_{\nu} u_{0}^{+}=0 \quad \text { on } \Omega_{0} \backslash\left(\Gamma_{0} \cup Q\right), \\
\left\{\begin{array}{l}
u_{0}^{(k)} \leq g_{k}, \quad a_{k}\left|B_{k}\right| \partial_{x_{3} x_{3}}^{2} u_{0}^{(k)}+\left|B_{k}\right| \mu_{k}\left(u_{0}^{(k)}\right) \leq-\delta_{\alpha_{k}, 1} l_{k} h_{k}\left(u_{0}^{(k)}\right), \\
\left(u_{0}^{(k)}-g_{k}\right)\left(-a_{k}\left|B_{k}\right| \partial_{x_{3} x_{3}}^{2} u_{0}^{(k)}+\left|B_{k}\right| \mu_{k}\left(u_{0}^{(k)}\right)+\delta_{\alpha_{k}, 1} l_{k} h_{k}\left(u_{0}^{(k)}\right)\right)=0,
\end{array}\right. \\
\left.u_{0}^{+}\right|_{x_{3}=0}=\left.u_{0}^{(k)}\right|_{x_{3}=0}, \quad k=1, \ldots, m, \\
\partial_{x_{3}} u_{0}^{+}\left(x^{\prime}, 0\right)=\sum_{k=1}^{K_{0}} a_{k}\left|B_{k}\right| \partial_{x_{3}} u_{0}^{(k)}\left(x^{\prime}, 0\right), \quad\left(x^{\prime}, 0\right) \in Q, \\
\left.\partial_{x_{3}} u_{0}^{(k)}\right|_{x_{3}=-d_{k}}=0, \quad k=1, \ldots, m,
\end{array}\right.
$$

which is called the homogenized problem for the problem (1.2)-(1.3).

\subsection{Existence and uniqueness of a weak solution to the homogenized problem}

We introduce the space $\mathcal{V}_{0}:=L^{2}\left(\Omega_{0}\right) \times L^{2}\left(D_{1}\right) \times \cdots \times L^{2}\left(D_{m}\right)$ with the scalar product

$$
(\mathbf{u}, \mathbf{v}) \mathcal{V}_{0}=\int_{\Omega_{0}} u_{0} v_{0} d x+\sum_{k=1}^{m} \int_{D_{k}} u_{k} v_{k} d x
$$

where $\mathbf{u}=\left(u_{0}, u_{1}, \ldots, u_{m}\right)$ and $\mathbf{v}=\left(v_{0}, v_{1}, \ldots, v_{m}\right)$. Also we define the Hilbert space

$$
\begin{aligned}
\mathcal{H}_{0}:= & \left\{\mathbf{u} \in \mathcal{V}_{0}: u_{0} \in H^{1}\left(\Omega_{0} ; \Gamma_{0}\right), u_{k} \in W^{0,0,1}\left(D_{k}\right)\right. \text { and } \\
& \left.u_{0}^{+}\left(x^{\prime}, 0\right)=u_{k}\left(x^{\prime}, 0\right) \text { for a.e. } x^{\prime} \in Q, k=1, \ldots, m\right\}
\end{aligned}
$$

with the scalar product

$$
(\mathbf{u}, \mathbf{v})_{\mathcal{H}_{0}}=\int_{\Omega_{0}} \nabla u_{0}^{+} \cdot \nabla v_{0}^{+} d x+\sum_{k=1}^{m} \int_{D_{k}}\left(u_{k} v_{k}+\partial_{x_{3}} u_{k} \partial_{x_{3}} v_{k}\right) d x
$$

Define also the subset

$$
\mathcal{K}_{0}=\left\{\boldsymbol{\varphi} \in \mathcal{H}_{0}: \varphi_{k} \leq g_{k} \text { a.e. in } D_{k}, k=1, \ldots, m\right\}
$$

It is obvious that $\mathcal{K}_{0}$ is a closed and convex in $\mathcal{H}_{0}$. 
The homogenized problem (3.4) is associated with the operator $\mathcal{A}: \mathcal{H}_{0} \mapsto \mathcal{H}_{0}^{*}$

$$
\begin{aligned}
\langle\mathcal{A} \mathbf{u}, \mathbf{v}\rangle_{0}:= & \int_{\Omega_{0}} \nabla u_{0} \cdot \nabla v_{0} d x+\sum_{k=1}^{m} a_{k}\left|B_{k}\right| \int_{D_{k}} \partial_{x_{3}} u_{k} \partial_{x_{3}} v_{k} d x+\int_{\Omega_{0}} \mu_{0}\left(u_{0}\right) v_{0} d x \\
& +\sum_{k=1}^{m}\left|B_{k}\right| \int_{D_{k}} \mu_{k}\left(u_{k}\right) v_{k} d x+\sum_{k=1}^{m} \delta_{\alpha_{k}, 1} l_{k} \int_{D_{k}} h_{k}\left(u_{k}\right) v_{k} d x \quad \forall \mathbf{u}, \mathbf{v} \in \mathcal{H}_{0}
\end{aligned}
$$

where $\langle\cdot, \cdot\rangle_{0}$ is the duality pairing of $\mathcal{H}_{0}^{*}$ and $\mathcal{H}_{0}$.

With the help of (1.5)-(1.6) and similarly as in Section 2.1, we prove that the operator $\mathcal{A}$ is coercive, strongly monotone, bounded, and hemicontinuous.

By the same way as in Section 2 we can give equivalent definitions of a weak solution to the problem (3.4). For this purpose define the multi-valued function

$$
\mathbf{g}(x):= \begin{cases}0, & x \in \Omega_{0}, \\ g_{k}(x), & x \in D_{k}, k=1, \ldots, m .\end{cases}
$$

Obviously $\mathbf{g} \in \mathcal{K}_{0}$.

Definition 3.1 A function $\mathbf{u}_{0} \in \mathcal{K}_{0}$ is called a weak solution to problem (3.4) if it satisfies the equality

$$
\left\langle\mathcal{A} \mathbf{u}_{0}, \mathbf{u}_{0}-\mathbf{g}\right\rangle_{0}=\left\langle F, \mathbf{u}_{0}\right\rangle_{0}
$$

and inequality

$$
\left\langle\mathcal{A} \mathbf{u}_{0}, \boldsymbol{\varphi}-\mathbf{g}\right\rangle_{0} \geq\langle F, \boldsymbol{\varphi}\rangle_{0} \quad \forall \boldsymbol{\varphi} \in \mathcal{K}_{0}
$$

where the linear functional $F \in \mathcal{H}_{0}^{*}$ is defined as follows:

$$
\langle F, \mathbf{v}\rangle_{0}:=\int_{\Omega_{0}} f v_{0} d x \quad \forall \mathbf{v} \in \mathcal{H}_{0}
$$

Definition 3.2 A function $\mathbf{u}_{0} \in \mathcal{K}_{0}$ is called a weak solution to problem (3.4) if it satisfies the inequality

$$
\left\langle\mathcal{A} \mathbf{u}_{0}, \boldsymbol{\varphi}-\mathbf{u}_{0}\right\rangle_{0} \geq\left\langle F, \boldsymbol{\varphi}-\mathbf{u}_{0}\right\rangle_{0} \quad \forall \boldsymbol{\varphi} \in \mathcal{K}_{0}
$$

Due to the inequality

$$
\begin{aligned}
\int_{\Omega_{0}} \mid & \left.\nabla\left(\varphi-u_{0}^{+}\right)\right|^{2} d x+\sum_{k=1}^{m} a_{k}\left|B_{k}\right| \int_{D_{k}}\left|\partial_{x_{3}}\left(\varphi_{k}-u_{0}^{(k)}\right)\right|^{2} d x \\
& +\int_{\Omega_{0}}\left(\mu_{0}\left(\varphi_{0}\right)-\mu_{0}\left(u_{0}^{+}\right)\right)\left(\varphi_{0}-u_{0}^{+}\right) d x \\
& +\sum_{k=1}^{m}\left|B_{k}\right| \int_{D_{k}}\left(\mu_{k}\left(\varphi_{k}\right)-\mu_{k}\left(u_{0}^{(k)}\right)\right)\left(\varphi_{k}-u_{0}^{(k)}\right) d x
\end{aligned}
$$




$$
\begin{aligned}
& \quad+\sum_{k=1}^{m} \delta_{\alpha_{k}, 1} \int_{D_{k}} l_{k}\left(h_{k}\left(\varphi_{k}\right)-h_{k}\left(u_{0}^{(k)}\right)\right)\left(\varphi_{k}-u_{0}^{(k)}\right) d x \\
& \geq 0 \quad \forall \varphi \in \mathcal{K}_{0},
\end{aligned}
$$

which is valid because of (1.5)-(1.6), we can give another equivalent definition.

Definition 3.3 A function $\mathbf{u}_{0} \in \mathcal{K}_{0}$ is called a weak solution to problem (3.4) if it satisfies the equality

$$
\left\langle\mathcal{A} \varphi, \varphi-\mathbf{u}_{0}\right\rangle_{0} \geq\left\langle F, \varphi-\mathbf{u}_{0}\right\rangle_{0} \quad \forall \varphi \in \mathcal{K}_{0} .
$$

Let us show that these definitions are equivalent. Setting the arbitrary multi-valued function $\varphi=\mathbf{u}_{0}+t\left(\psi-\mathbf{u}_{0}\right) \in \mathcal{K}_{0}\left(t \in[0,1], \psi \in \mathcal{K}_{0}\right)$ in inequality (3.7), we get

$$
\left\langle\mathcal{A}\left(\mathbf{u}_{0}+t\left(\boldsymbol{\psi}-\mathbf{u}_{0}\right)\right), \boldsymbol{\psi}-\mathbf{u}_{0}\right\rangle_{0} \geq\left\langle F, \boldsymbol{\psi}-\mathbf{u}_{0}\right\rangle_{0}
$$

from which, using hemicontinuousness of the operator $\mathcal{A}$, we obtain (3.5). Adding the inequality (3.6) to (3.5), we get (3.7).

Thus, by virtue of properties of the operator $\mathcal{A}$ and Theorems 8.2, 8.3 from [7], the homogenized problem (3.4) has the unique weak solution.

\subsection{The proof of the convergence theorem}

From (2.17) it follows that the values $\left\|u_{\varepsilon}\right\|_{H^{1}\left(\Omega_{0}\right)},\left\|{\widetilde{u_{\varepsilon}}}^{(k)}\right\|_{L^{2}\left(D_{k}\right)},\left\|\widetilde{\partial_{x_{i}} u_{\varepsilon}}{ }^{(k)}\right\|_{L^{2}\left(D_{k}\right)}(i=1,2,3$, $k=1, \ldots, m)$ are uniformly bounded with respect to $\varepsilon$. Therefore we can choose a subsequence $\left\{\varepsilon^{\prime}\right\} \subset\{\varepsilon\}$ (again denoted by $\varepsilon$ ) such that

$$
\left.\begin{array}{ll}
\left.u_{\varepsilon}\right|_{\Omega_{0}} \stackrel{w}{\longrightarrow} u_{0}^{+} & \text {weakly in } H^{1}\left(\Omega_{0}\right), \\
\widetilde{u_{\varepsilon}}(k) \stackrel{w}{\longrightarrow}\left|B_{k}\right|\left(\left|B_{k}\right|^{-1} u^{(k)}\right)=:\left|B_{k}\right| u_{0}^{(k)} & \text { weakly in } L^{2}\left(D_{k}\right), \\
\widetilde{\partial_{x_{i}} u_{\varepsilon}} \stackrel{\text { we }}{\longrightarrow} \gamma_{i}^{(k)} & \text { weakly in } L^{2}\left(D_{k}\right),
\end{array}\right\} \quad \text { as } \varepsilon \rightarrow 0
$$

where $u_{0}^{+}, u_{0}^{(k)}, \gamma_{i}^{(k)}, k=1, \ldots, m, i=1,2,3$ are some functions that will be determined in the following.

1. At first we determine functions $\gamma_{i}^{(k)}, i=1,2,3, k=1, \ldots, m$. Consider an arbitrary function $\psi \in C_{0}^{\infty}\left(D_{k}\right)$. Since $\partial_{x_{3}}\left({\widetilde{u_{\varepsilon}}}^{(k)}\right)=\widetilde{\partial_{x_{3}} u_{\varepsilon}}(k)$,

$$
\int_{D_{k}} \widetilde{\partial_{x_{3}} u_{\varepsilon}}{ }^{(k)} \psi d x=-\int_{D_{k}}{\widetilde{u_{\varepsilon}}}^{(k)} \partial_{x_{3}} \psi d x \quad \forall \psi \in C_{0}^{\infty}\left(D_{k}\right)
$$

Passing to the limit as $\varepsilon \rightarrow 0$ in this identities, we obtain

$$
\int_{D_{k}} \gamma_{3}^{(k)} \psi d x=-\left|B_{k}\right| \int_{D_{k}} u_{0}^{(k)} \partial_{x_{3}} \psi d x \quad \forall \psi \in C_{0}^{\infty}\left(D_{k}\right)
$$

whence it follows that there exist a weak derivative $\partial_{x_{3}} u_{0}^{(k)}$ and $\gamma_{3}^{(k)}=\left|B_{k}\right| \partial_{x_{3}} u_{0}^{(k)}$ a.e. in $D_{k}$, $k=1, \ldots, m$. 
Consider the functions $Z_{j}\left(\xi_{j}\right)=-\xi_{j}+\left[\xi_{j}\right], j=1,2$, where $[t]$ is the integer part of $t$. With the help of these functions we determine for every $k_{0} \in\{1, \ldots, m\}$ the following test-functions:

$$
\Phi_{j}^{\left(k_{0}\right)}(x)=\left\{\begin{array}{ll}
0, & x \in \Omega_{0}, \\
\varepsilon Z_{j}\left(\frac{x_{j}}{\varepsilon}\right) \psi_{k_{0}}(x)+g_{k_{0}}(x), & x \in G_{\varepsilon}\left(k_{0}\right), \\
g_{k}(x), & x \in G_{\varepsilon}(k), k \in\{1, \ldots, m\} \backslash\left\{k_{0}\right\},
\end{array} \quad j=1,2,\right.
$$

where $\psi_{k_{0}}$ is arbitrary positive function from $C_{0}^{\infty}\left(D_{k_{0}}\right)$.

Since $Z_{j} \leq 0$ and $\psi_{k_{0}} \geq 0$, the functions $\left\{\Phi_{1}^{\left(k_{0}\right)}, \Phi_{2}^{\left(k_{0}\right)}\right\}_{k_{0}=1}^{m}$ belong to $\mathcal{K}_{\varepsilon}$ and

$$
\begin{aligned}
& \Phi_{j}^{\left(k_{0}\right)}(x)-\mathcal{G}(x)= \begin{cases}0, & x \in \Omega_{\varepsilon} \backslash G_{\varepsilon}\left(k_{0}\right), \quad j=1,2, \\
\varepsilon Z_{j}\left(\frac{x_{j}}{\varepsilon}\right) \psi_{k_{0}}(x), & x \in G_{\varepsilon}\left(k_{0}\right),\end{cases} \\
& \nabla\left(\Phi_{1}^{\left(k_{0}\right)}-g_{k_{0}}\right)=\left(-\psi_{k_{0}}, 0,0\right)+\varepsilon Z_{1}\left(\frac{x_{1}}{\varepsilon}\right) \nabla \psi_{k_{0}} \quad \text { in } G_{\varepsilon}\left(k_{0}\right), \\
& \nabla\left(\Phi_{2}^{\left(k_{0}\right)}-g_{k_{0}}\right)=\left(0,-\psi_{k_{0}}, 0\right)+\varepsilon Z_{2}\left(\frac{x_{2}}{\varepsilon}\right) \nabla \psi_{k_{0}} \quad \text { in } G_{\varepsilon}\left(k_{0}\right) .
\end{aligned}
$$

Substituting the functions $\left\{\Phi_{j}^{\left(k_{0}\right)}\right\}$ into the integral inequality (2.5), we get

$$
\begin{aligned}
& -a_{k_{0}} \int_{G_{\varepsilon}\left(k_{0}\right)} \partial_{x_{j}} u_{\varepsilon} \psi_{k_{0}} d x+\varepsilon a_{k_{0}} \int_{G_{\varepsilon}\left(k_{0}\right)} Z_{j}\left(\frac{x_{j}}{\varepsilon}\right) \nabla u_{\varepsilon} \cdot \nabla \psi_{k_{0}} d x \\
& \quad+\varepsilon \int_{G_{\varepsilon}\left(k_{0}\right)} \mu_{k_{0}}\left(u_{\varepsilon}\right) Z_{j}\left(\frac{x_{j}}{\varepsilon}\right) \psi_{k_{0}} d x \\
& \geq-\varepsilon^{\alpha_{k_{0}}+1} \int_{S_{\varepsilon}\left(k_{0}\right)} h_{k_{0}}\left(u_{\varepsilon}\right) Z_{j}\left(\frac{x_{j}}{\varepsilon}\right) \psi_{k_{0}} d \sigma_{x} .
\end{aligned}
$$

Owing to (1.5), (1.6), (2.13), and (2.17), we deduce from (3.11) the estimate

$$
\left|\int_{D_{k_{0}}} \widetilde{\partial_{x_{j}} u_{\varepsilon}}{ }^{\left(k_{0}\right)} \psi_{k_{0}} d x\right| \leq C_{1} \varepsilon
$$

whence we get $\gamma_{1}^{\left(k_{0}\right)}=\gamma_{2}^{\left(k_{0}\right)}=0$ a.e. in $D_{k_{0}}, k_{0}=1, \ldots, m$.

2. By virtue of the continuity of the trace operator, the compact embedding $H^{1 / 2}(Q) \subset$ $L^{2}(Q)$, and the first relation in (3.8), we have

$$
u_{\varepsilon}\left(x^{\prime}, 0+0\right) \rightarrow u_{0}^{+}\left(x^{\prime}, 0\right) \quad \text { strongly in } L^{2}(Q) \text { as } \varepsilon \rightarrow 0 .
$$

Consider 1-periodic functions

$$
\chi_{k}\left(\xi^{\prime}\right)=\left\{\begin{array}{l}
1, \xi^{\prime} \in B_{k}, \\
0, \xi^{\prime} \in \square \backslash B_{k}, \quad k=1, \ldots, m .
\end{array}\right.
$$

It is known that

$$
\chi_{k}\left(\frac{x^{\prime}}{\varepsilon}\right) \stackrel{w}{\longrightarrow}\left|B_{k}\right| \quad \text { weakly in } L^{2}(Q) \text { as } \varepsilon \rightarrow 0 .
$$


Since ${\tilde{u_{\varepsilon}}}^{(k)}\left(x^{\prime}, 0-0\right)=\chi_{k}\left(\frac{x^{\prime}}{\varepsilon}\right) u_{\varepsilon}\left(x^{\prime}, 0+0\right)$ a.e. in $Q$,

$$
\tilde{u}_{\varepsilon}^{(k)}\left(x^{\prime}, 0-0\right) \stackrel{w}{\longrightarrow}\left|B_{k}\right| u_{0}^{+}\left(x^{\prime}, 0\right) \quad \text { weakly in } L^{2}(Q) \text { as } \varepsilon \rightarrow 0 .
$$

On the other hand,

$$
\int_{Q}{\tilde{u_{\varepsilon}}}^{(k)}\left(x^{\prime}, 0\right) \psi\left(x^{\prime}\right) d x^{\prime}=\frac{1}{d_{k}} \int_{D_{k}}\left(\tilde{u}_{\varepsilon}^{(k)}(x) \psi\left(x^{\prime}\right)+\left(x_{3}+d_{k}\right) \partial_{x_{3}}{\tilde{u_{\varepsilon}}}^{(k)}(x) \psi\left(x^{\prime}\right)\right) d x
$$

for any function $\psi \in C_{0}^{\infty}(Q)$ and $k \in\{1, \ldots, m\}$. Passing to the limit in this equality and taking into account the second relation in (3.8) and the convergence results obtained above, we obtain

$$
\left|B_{k}\right| \int_{Q} u_{0}^{+}\left(x^{\prime}, 0\right) \psi\left(x^{\prime}\right) d x^{\prime}=\frac{\left|B_{k}\right|}{d_{k}} \int_{D_{k}}\left(u_{0}^{(k)}(x) \psi\left(x^{\prime}\right)+\left(x_{3}+d_{k}\right) \partial_{x_{3}} u_{0}^{(k)}(x) \psi\left(x^{\prime}\right)\right) d x,
$$

whence

$$
u_{0}^{+}\left(x^{\prime}, 0\right)=u_{0}^{(k)}\left(x^{\prime}, 0\right) \quad \text { for a.e. } x^{\prime} \in Q, k=1, \ldots, m \text {. }
$$

3. Let us show that

$$
u_{0}^{(k)} \leq g_{k} \quad \text { a.e. in } D_{k}, k=1, \ldots, m \text {. }
$$

For this purpose we take any nonnegative function $\phi \in C^{\infty}\left(\overline{D_{k}}\right)$ and insert $u_{\varepsilon} \phi$ into (2.10) instead of $v$. Since $\phi \geq 0$ and $u_{\varepsilon} \in \mathcal{K}_{\varepsilon}$, we get

$$
\begin{aligned}
& \frac{l_{k}}{\left|B_{k}\right|} \int_{D_{k}} \tilde{u_{\varepsilon}}{ }^{(k)} \phi d x+\left.\varepsilon \int_{G_{\varepsilon}(k)} \nabla_{\xi^{\prime}} Y_{k}\left(\xi^{\prime}\right)\right|_{\xi^{\prime}=\frac{x^{\prime}}{\varepsilon}} \cdot \nabla_{x^{\prime}}\left(u_{\varepsilon} \phi\right) d x \\
& \quad \leq \varepsilon \int_{S_{\varepsilon}(k)} g_{k} \phi d \sigma_{x} \\
& \quad=\frac{l_{k}}{\left|B_{k}\right|} \int_{D_{k}} \chi_{k}\left(\frac{x^{\prime}}{\varepsilon}\right) g_{k} \phi d x+\left.\varepsilon \int_{G_{\varepsilon}(k)} \nabla_{\xi^{\prime}} Y_{k}\left(\xi^{\prime}\right)\right|_{\xi^{\prime}=\frac{x^{\prime}}{\varepsilon}} \cdot \nabla_{x^{\prime}}\left(g_{k} \phi\right) d x .
\end{aligned}
$$

Taking into account (2.17), the second limit in (3.8) and (2.12), we can pass to the limit in $(3.14)$ as $\varepsilon \rightarrow 0$. As a result, we have

$$
\int_{D_{k}} u_{0}^{(k)} \phi d x \leq \int_{D_{k}} g_{k} \phi d x \quad \forall \phi \in C^{\infty}\left(\overline{D_{k}}\right), \phi \geq 0,
$$

whence (3.13) follows.

4. Consider the following set of multi-valued functions:

$$
\begin{aligned}
C_{\mathcal{G}}^{\infty}\left(\Omega_{0}, D_{1}, \ldots, D_{m}\right):= & \left\{\left(\varphi_{0}, \varphi_{1}, \ldots, \varphi_{m}\right): \varphi_{0} \in C^{\infty}\left(\overline{\Omega_{0}}\right),\left.\varphi_{0}\right|_{\Gamma_{0}}=0,\right. \\
& \varphi_{k} \in C^{\infty}\left(\overline{D_{k}}\right), \varphi_{k} \leq g_{k} \text { in } D_{k}, k=1, \ldots, m, \\
& \left.\varphi_{0}^{+}\left(x^{\prime}, 0\right)=\varphi_{k}\left(x^{\prime}, 0\right) \text { for } x^{\prime} \in Q, k=1, \ldots, m\right\} .
\end{aligned}
$$

Obviously, for all $\varphi \in C_{\mathcal{G}}^{\infty}\left(\Omega_{0}, D_{1}, \ldots, D_{m}\right)$ its restriction $\left(\varphi_{0},\left.\varphi_{1}\right|_{G_{\varepsilon}(1)}, \ldots,\left.\varphi_{m}\right|_{G_{\varepsilon}(m)}\right)$ belongs to $\mathcal{K}_{\varepsilon}$. 
Now let us add to inequality (2.9) the inequality

$$
\begin{aligned}
\int_{\Omega_{0}} \mid & \left.\nabla\left(\varphi-u_{\varepsilon}\right)\right|^{2} d x+\sum_{k=1}^{m} a_{k} \int_{G_{\varepsilon}(k)}\left|\partial_{x_{3}}\left(\varphi-u_{\varepsilon}\right)\right|^{2} d x+\sum_{k=1}^{m} a_{k} \int_{G_{\varepsilon}(k)}\left|\partial_{x_{1}} u_{\varepsilon}\right|^{2} d x \\
& +\sum_{k=1}^{m} a_{k} \int_{G_{\varepsilon}(k)}\left|\partial_{x_{2}} u_{\varepsilon}\right|^{2} d x+\int_{\Omega_{0}}\left(\mu_{0}(\varphi)-\mu_{0}\left(u_{\varepsilon}\right)\right)\left(\varphi-u_{\varepsilon}\right) d x \\
& +\sum_{k=1}^{m} \int_{G_{\varepsilon}(k)}\left(\mu_{k}(\varphi)-\mu_{k}\left(u_{\varepsilon}\right)\right)\left(\varphi-u_{\varepsilon}\right) d x \\
& +\sum_{k=1}^{m} \varepsilon^{\alpha_{k}} \int_{S_{\varepsilon}(k)}\left(h_{k}(\varphi)-h_{k}\left(u_{\varepsilon}\right)\right)\left(\varphi-u_{\varepsilon}\right) d \sigma_{x} \\
\geq & 0,
\end{aligned}
$$

where $\varphi$ is arbitrary function from $C_{\mathcal{G}}^{\infty}\left(\Omega_{0}, D_{1}, \ldots, D_{m}\right)$. We get

$$
\begin{aligned}
& \int_{\Omega_{0}} \nabla \varphi \cdot \nabla\left(\varphi-u_{\varepsilon}\right) d x+\sum_{k=1}^{m} a_{k} \int_{G_{\varepsilon}(k)} \partial_{x_{1}} u_{\varepsilon} \partial_{x_{1}} \varphi d x+\sum_{k=1}^{m} a_{k} \int_{G_{\varepsilon}(k)} \partial_{x_{2}} u_{\varepsilon} \partial_{x_{2}} \varphi d x \\
& \quad+\sum_{k=1}^{m} a_{k} \int_{G_{\varepsilon}(k)} \partial_{x_{3}} \varphi \partial_{x_{3}}\left(\varphi-u_{\varepsilon}\right) d x+\int_{\Omega_{0}} \mu_{0}(\varphi)\left(\varphi-u_{\varepsilon}\right) d x \\
& \quad+\sum_{k=1}^{m} \int_{G_{\varepsilon}(k)} \mu_{k}(\varphi)\left(\varphi-u_{\varepsilon}\right) d x+\sum_{k=1}^{m} \varepsilon^{\alpha_{k}} \int_{S_{\varepsilon}(k)} h_{k}(\varphi)\left(\varphi-u_{\varepsilon}\right) d \sigma_{x} \\
& \quad \geq \int_{\Omega_{0}} f\left(\varphi-u_{\varepsilon}\right) d x,
\end{aligned}
$$

which with the help of (2.10) we can rewrite as

$$
\begin{aligned}
& \int_{\Omega_{0}} \nabla \varphi_{0} \cdot \nabla\left(\varphi_{0}-u_{\varepsilon}\right) d x+\sum_{k=1}^{m} a_{k} \int_{D_{k}} \widetilde{\partial_{x_{1}} u_{\varepsilon}^{(k)}} \partial_{x_{1}} \varphi_{k} d x+\sum_{k=1}^{m} a_{k} \int \widetilde{\partial_{D_{k}} u_{\varepsilon}^{(k)}} \partial_{x_{2}} \varphi_{k} d x
\end{aligned}
$$

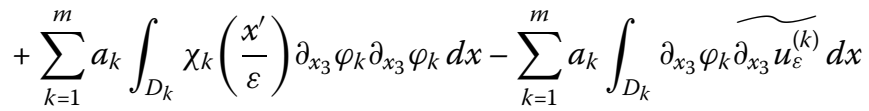

$$
\begin{aligned}
& +\int_{\Omega_{0}} \mu_{0}\left(\varphi_{0}\right)\left(\varphi_{0}-u_{\varepsilon}\right) d x+\sum_{k=1}^{m} \int_{D_{k}} \chi_{k}\left(\frac{x^{\prime}}{\varepsilon}\right) \mu_{k}\left(\varphi_{k}\right) \varphi_{k} d x-\sum_{k=1}^{m} \int_{D_{k}} \mu_{k}\left(\varphi_{k}\right) \tilde{u}_{\varepsilon}^{(k)} d x \\
& +\sum_{k=1}^{m} \varepsilon^{\alpha_{k}-1} \frac{l_{k}}{\left|B_{k}\right|} \int_{D_{k}} \chi_{k}\left(\frac{x^{\prime}}{\varepsilon}\right) h_{k}\left(\varphi_{k}\right) \varphi_{k} d x-\sum_{k=1}^{m} \varepsilon^{\alpha_{k}-1} \frac{l_{k}}{\left|B_{k}\right|} \int_{D_{k}} h_{k}\left(\varphi_{k}\right) \tilde{u}_{\varepsilon}^{(k)} d x \\
& +\left.\sum_{k=1}^{m} \varepsilon^{\alpha_{k}} \int_{D_{k}} \chi_{k}\left(\frac{x^{\prime}}{\varepsilon}\right) \nabla_{\xi^{\prime}} Y_{k}\left(\xi^{\prime}\right)\right|_{\xi^{\prime}=\frac{x^{\prime}}{\varepsilon}} \cdot \nabla_{x^{\prime}}\left(\varphi_{k} h_{k}\left(\varphi_{k}\right)\right) d x \\
& -\left.\sum_{k=1}^{m} \varepsilon^{\alpha_{k}} \int_{G_{\varepsilon}(k)} \nabla_{\xi^{\prime}} Y_{k}^{(m)}\left(\xi^{\prime}\right)\right|_{\xi^{\prime}=\frac{x^{\prime}}{\varepsilon}} \cdot \nabla_{x^{\prime}}\left(h_{k}\left(\varphi_{k}\right) u_{\varepsilon}\right) d x \\
& \geq \int_{\Omega_{0}} f\left(\varphi_{0}-u_{\varepsilon}\right) d x
\end{aligned}
$$


Taking into account (2.12), (2.17), (3.12), the convergence results (3.2) obtained in the first item of the proof, and the assumption that $\alpha_{k} \geq 1$, we can pass to the limit in (3.17) as $\varepsilon \rightarrow 0$. As a result, we obtain the inequality

$$
\begin{aligned}
& \int_{\Omega_{0}} \nabla \varphi_{0} \cdot \nabla\left(\varphi_{0}-u_{0}^{+}\right) d x+\sum_{k=1}^{m} a_{k}\left|B_{k}\right| \int_{D_{k}} \partial_{x_{3}} \varphi_{k} \partial_{x_{3}}\left(\varphi_{k}-u_{0}^{(k)}\right) d x \\
& \quad+\int_{\Omega_{0}} \mu_{0}\left(\varphi_{0}\right)\left(\varphi_{0}-u_{0}^{+}\right) d x+\sum_{k=1}^{m}\left|B_{k}\right| \int_{D_{k}} \mu_{k}\left(\varphi_{k}\right)\left(\varphi_{k}-u_{0}^{(k)}\right) d x \\
& \quad+\sum_{k=1}^{m} \delta_{\alpha_{k}, 1} l_{k} \int_{D_{k}} h_{k}\left(\varphi_{k}\right)\left(\varphi_{k}-u_{0}^{(k)}\right) d x \\
& \geq \int_{\Omega_{0}} f\left(\varphi_{0}-u_{0}^{+}\right) d x \quad \forall \varphi \in C_{\mathcal{G}}^{\infty}\left(\Omega_{0}, D_{1}, \ldots, D_{m}\right) .
\end{aligned}
$$

Since $C_{\mathcal{G}}^{\infty}\left(\Omega_{0}, D_{1}, \ldots, D_{m}\right)$ is dense in $\mathcal{K}_{0}$, the integral inequality (3.18) is valid for any multi-valued function $\varphi \in \mathcal{K}_{0}$. This and inequalities (3.13) mean that the multi-valued function $\mathbf{u}_{0}$ defined by (3.3) is the unique solution of inequality (3.7) (see Definition 3.3) and, moreover, it is the weak solution to the homogenized problem (3.4). Owing to the uniqueness of this solution, the above argumentations are true for any subsequence of $\{\varepsilon\}$ chosen at the beginning of the proof. Thus the limits in (3.2) hold.

\section{Convergence theorem in the case $\alpha_{k_{0}}<1$}

Now let us suppose that for some $k=k_{0}$ parameter $\alpha_{k_{0}}<1$ and the other parameters $\left\{\alpha_{k}\right\}$ are greater than or equal to one; for definiteness let $k_{0}=1$, i.e., $\alpha_{1}<1, \alpha_{k} \geq 1, k=2, \ldots, m$. In this case we additionally assume that the condition $\mathrm{C} 3$ is satisfied.

Theorem 4.1 (Case $\left.\alpha_{1}<1, \alpha_{k} \geq 1, k=2, \ldots, n\right)$ Let the conditions described above are performed. Then the sequence of solutions $\left\{u_{\varepsilon}\right\}_{\varepsilon>0}$ to the problem (1.2)-(1.3) satisfies the relations

$$
\left.\begin{array}{ll}
\left.u_{\varepsilon}\right|_{\Omega_{0}} \stackrel{w}{\rightarrow} u_{0}^{+} & \text {weakly in } H^{1}\left(\Omega_{0} ; \Gamma_{0}\right), \\
{\tilde{u_{\varepsilon}}}^{(1)} \stackrel{s}{\longrightarrow} 0 & \text { strongly in } L^{2}\left(D_{1}\right), \\
\widetilde{u_{\varepsilon}}(k) \stackrel{w}{\longrightarrow}\left|B_{k}\right| u_{0}^{(k)} & \text { weakly in } W^{0,0,1}\left(D_{k}\right), \\
\widetilde{\partial_{x_{i}} u_{\varepsilon}} \stackrel{w}{\longrightarrow} 0 & \text { weakly in } L^{2}\left(D_{k}\right), i=1,2
\end{array}\right\} \text { as } \varepsilon \rightarrow 0
$$

for $k=2, \ldots, m$, where $u_{0}^{+}$is the unique weak solution to the following problem:

$$
\left\{\begin{array}{l}
-\Delta_{x} u_{0}^{+}+\mu_{0}\left(u_{0}^{+}\right)=f \quad \text { in } \Omega_{0}, \\
u_{0}^{+}=0 \quad \text { on } \Gamma_{0} \cup Q, \quad \text { and } \quad \partial_{\nu} u_{0}^{+}=0 \quad \text { on } \partial \Omega_{0} \backslash\left(\Gamma_{0} \cup Q\right),
\end{array}\right.
$$

and for every $k \in\{2, \ldots, m\}$ the function $u_{0}^{(k)}$ is the unique weak solution of the following problem:

$$
\left\{\begin{array}{l}
\left\{\begin{array}{l}
u_{0}^{(k)} \leq g_{k}, \quad-a_{k}\left|B_{k}\right| \partial_{x_{3} x_{3}}^{2} u_{0}^{(k)}+\left|B_{k}\right| \mu_{k}\left(u_{0}^{(k)}\right) \leq-\delta_{\alpha_{k}, 1} l_{k} h_{k}\left(u_{0}^{(k)}\right), \\
\left(u_{0}^{(k)}-g_{k}\right)\left(-a_{k}\left|B_{k}\right| \partial_{x_{3} x_{3}}^{2} u_{0}^{(k)}+\left|B_{k}\right| \mu_{k}\left(u_{0}^{(k)}\right)+\delta_{\alpha_{k}, 1} l_{k} h_{k}\left(u_{0}^{(k)}\right)\right)=0,
\end{array} \quad \text { in } D_{k},\right. \\
\left.u_{0}^{(k)}\right|_{x_{3}=0}=0,\left.\quad \partial_{x_{3}} u_{0}^{(k)}\right|_{x_{3}=-d_{k}}=0 .
\end{array}\right.
$$




\subsection{Weak solutions of problems (4.2) and (4.3)}

Definition 4.1 A function $u_{0}^{+} \in H^{1}\left(\Omega_{0} ; \Gamma_{0} \cup Q\right)$ is called a weak solution to the problem (4.2) if it satisfies the integral identity

$$
\int_{\Omega_{0}} \nabla u_{0}^{+} \cdot \nabla \varphi d x+\int_{\Omega_{0}} \mu_{0}\left(u_{0}^{+}\right) v d x \quad \forall \varphi \in H^{1}\left(\Omega_{0} ; \Gamma_{0} \cup Q\right),
$$

where $H^{1}\left(\Omega_{0} ; \Gamma_{0} \cup Q\right):=\left\{u \in H^{1}\left(\Omega_{0}\right):\left.u\right|_{\Gamma_{0} \cup Q}=0\right\}$.

Due to the condition $\mathrm{C} 2$, it follows from the main results of the theory of semilinear boundary-value problems that the problem (4.2) has the unique weak solution.

For the treatment of the problem (4.3) at fixed $k \in\{2, \ldots, m\}$ we introduce the anisotropic Sobolev space $W^{0,0,1}\left(D_{k} ; Q\right):=\left\{u \in W^{0,0,1}\left(D_{k}\right):\left.u\right|_{Q}=0\right\}$ and define an operator $\mathcal{A}_{k}$ : $W^{0,0,1}\left(D_{k} ; Q\right) \mapsto\left(W^{0,0,1}\left(D_{k} ; Q\right)\right)^{*}$ through the relation

$$
\left\langle\mathcal{A}_{k} u, v\right\rangle_{k}=a_{k}\left|B_{k}\right| \int_{D_{k}} \partial_{x_{3}} u \partial_{x_{3}} v d x+\left|B_{k}\right| \int_{D_{k}} \mu_{k}(u) v d x+\delta_{\alpha_{k}, 1} l_{k} \int_{D_{k}} h_{k}(u) v d x
$$

for any $u, v \in W^{0,0,1}\left(D_{k} ; Q\right)$, where $\langle\cdot, \cdot\rangle_{k}$ is pairing of $\left(W^{0,0,1}\left(D_{k} ; Q\right)\right)^{*}$ and $W^{0,0,1}\left(D_{k} ; Q\right)$.

Also we determine the subset

$$
\mathfrak{K}_{k}:=\left\{u \in W^{0,0,1}\left(D_{k} ; Q\right): u \leq g_{k} \text { a.e. in } D_{k}\right\} .
$$

Obviously $\mathfrak{K}_{k}$ is closed and convex in $W^{0,0,1}\left(D_{k} ; Q\right)$.

With the help of (1.5)-(1.6) and Cauchy's inequality with $\delta>0$, similarly to Section 2.1, we prove that the operator $\mathcal{A}_{k}$ is coercive, strong monotone, bounded, and hemicontinuous. Then, in the same way as in Section 2, we can give the definition of a weak solution to the problem (4.3) at any fixed index $k \in\{2, \ldots, m\}$.

Definition 4.2 A function $u_{0}^{(k)} \in \mathfrak{K}_{k}$ is called a weak solution to the problem (4.3) if it satisfies the inequality

$$
\left\langle\mathcal{A}_{k} \varphi, \varphi-u_{0}^{(k)}\right\rangle_{k} \geq 0 \quad \forall \varphi \in \mathfrak{K}_{k}
$$

Thanks to Theorems 8.2, 8.3 from [7], the problem (4.3) has a unique weak solution.

\subsection{The proof of Theorem 4.1}

1 . The convergences

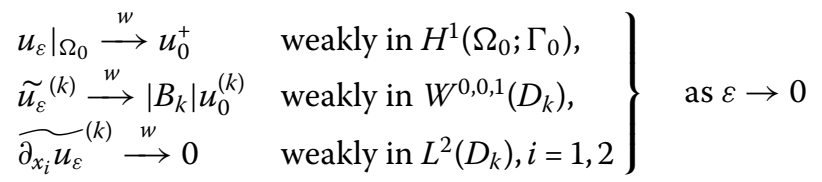

for every $k \in\{2, \ldots, m\}$ over a subsequence are proved in practically the same way as in the first item in the proof of Theorem 3.1.

2. We now show that the traces of the functions $u_{0}^{+}$and $\left\{u_{0}^{(k)}\right\}$ are equal to zero on $Q$. Using (1.5), (1.6), (2.17), and condition C3, we deduce from the integral identity (2.2) the 
following inequality:

$$
\begin{aligned}
& c_{1} \varepsilon^{\alpha_{1}} \int_{S_{\varepsilon}(1)} u_{\varepsilon}^{2} d \sigma_{x} \\
& \leq\|f\|_{L^{2}\left(\Omega_{0}\right)}\left\|u_{\varepsilon}\right\|_{L^{2}\left(\Omega_{0}\right)}+c_{5}\left\|u_{\varepsilon}\right\|_{\varepsilon}^{2}+\int_{\Omega_{0}}\left(\left|\mu_{0}(0)\right|+c_{3}\left|u_{\varepsilon}\right|\right)\left|u_{\varepsilon}\right| d x \\
& \quad+\sum_{k=1}^{m} \int_{G_{\varepsilon}(k)}\left(\left|\mu_{k}(0)\right|+c_{3}\left|u_{\varepsilon}\right|\right)\left|u_{\varepsilon}\right| d x+\sum_{k=2}^{m} \varepsilon^{\alpha_{k}} \int_{S_{\varepsilon}(k)}\left(\left|h_{k}(0)\right|+c_{3}\left|u_{\varepsilon}\right|\right)\left|u_{\varepsilon}\right| d \sigma_{x} \\
& \leq C_{1} .
\end{aligned}
$$

Then, with the help of (2.14) we get

$$
\int_{G_{\varepsilon}(1)} u_{\varepsilon}^{2} d x \leq C_{2}\left(\varepsilon^{2} \int_{G_{\varepsilon}(1)}\left|\nabla_{x^{\prime}} u_{\varepsilon}\right|^{2} d x+\varepsilon^{1-\alpha_{1}} \varepsilon^{\alpha_{1}} \int_{S_{\varepsilon}(1)} u_{\varepsilon}^{2} d \sigma_{x}\right) \leq C_{3} \varepsilon^{\theta}
$$

where $\theta:=\min \left(2 ; 1-\alpha_{1}\right)>0$. Therefore

$$
{\widetilde{u_{\varepsilon}}}^{(1)} \stackrel{s}{\longrightarrow} 0 \quad \text { strongly in } L^{2}\left(D_{1}\right) \text { as } \varepsilon \rightarrow 0 .
$$

Arguing as in the second item in the proof of Theorem 3.1, we obtain

$$
u_{0}^{+}\left(x^{\prime}, 0\right)=u_{0}^{(k)}\left(x^{\prime}, 0\right)=0 \quad \text { for a.e. } x^{\prime} \in Q, k=1, \ldots m .
$$

Also similarly to the proof of (3.13), we deduce that

$$
u_{0}^{(k)} \in \mathfrak{K}_{k}, \quad k=2, \ldots, m
$$

3. Consider the arbitrary function $\varphi_{0} \in C^{\infty}\left(\overline{\Omega_{0}}\right)$ such that $\left.\varphi_{0}\right|_{\Gamma_{0} \cup Q}=0$. Obviously, the function

$$
\phi_{\tau}(x)= \begin{cases}\tau \varphi_{0}(x), & x \in \Omega_{0}, \\ 0, & x \in G_{\varepsilon}(1), \\ g_{k}(x), & x \in G_{\varepsilon}(k), k=2, \ldots, m,\end{cases}
$$

where $\tau \in \mathbb{R}$, belongs to $\mathcal{K}_{\varepsilon}$. Writing the inequality (2.8) with $\varphi=\phi_{\tau}$, we have

$$
\tau \int_{\Omega_{0}} \nabla u_{\varepsilon} \cdot \nabla \varphi_{0} d x+\tau \int_{\Omega_{0}} \mu_{0}\left(u_{\varepsilon}\right) \varphi_{0} d x \geq \tau \int_{\Omega_{0}} f \varphi_{0} d x .
$$

Replacing $\tau$ with $-\tau$, we deduce that in fact equality holds above, i.e.,

$$
\int_{\Omega_{0}} \nabla u_{\varepsilon} \cdot \nabla \varphi_{0} d x+\int_{\Omega_{0}} \mu_{0}\left(u_{\varepsilon}\right) \varphi_{0} d x=\int_{\Omega_{0}} f \varphi_{0} d x
$$

Since $\left.u_{\varepsilon}\right|_{\Omega_{0}} \stackrel{w}{\longrightarrow} u_{0}^{+}$weakly in $H^{1}\left(\Omega_{0} ; \Gamma_{0}\right)$ and strongly in $L^{2}\left(\Omega_{0}\right)$, we can pass to the limit over a subsequence of the sequence $\{\varepsilon\}$ in (4.8) and arrive at the identity

$$
\int_{\Omega_{0}} \nabla u_{0}^{+} \cdot \nabla \varphi_{0} d x+\int_{\Omega_{0}} \mu_{0}\left(u_{0}^{+}\right) \varphi_{0} d x=\int_{\Omega_{0}} f \varphi_{0} d x, \quad \forall \varphi_{0} \in C^{\infty}\left(\overline{\Omega_{0}}\right),\left.\varphi_{0}\right|_{\Gamma_{0}} \cup Q=0 .
$$


Hence, with regard to (4.6), $u_{0}^{+}$is indeed the unique weak solution to the problem (4.2).

4. Denote by $C_{\mathcal{G}, 0}^{\infty}\left(\Omega_{0}, D_{1}, \ldots, D_{m} ; Q\right)$ the subset of $C_{\mathcal{G}}^{\infty}\left(\Omega_{0}, D_{1}, \ldots, D_{m}\right)$ (see (3.15)) having the property that $\varphi_{1} \equiv 0$ (recall that $g_{1} \equiv 0$ ). Obviously

$$
\varphi_{0}^{+}\left(x^{\prime}, 0\right)=\varphi_{k}\left(x^{\prime}, 0\right)=0, \quad x^{\prime} \in Q, k=2, \ldots, m,
$$

for every $\varphi \in C_{\mathcal{G}, 0}^{\infty}\left(\Omega_{0}, D_{1}, \ldots, D_{m} ; Q\right)$.

Inserting the restriction $\left(\varphi_{0},\left.0\right|_{G_{\varepsilon}(1)}, \ldots,\left.\varphi_{m}\right|_{G_{\varepsilon}(m)}\right)$ of $\varphi \in C_{\mathcal{G}, 0}^{\infty}\left(\Omega_{0}, D_{1}, \ldots, D_{m} ; Q\right)$ in inequality (3.16) and then passing to the limit as $\varepsilon \rightarrow 0$ similarly to the fourth item of the proof of Theorem 3.1, we find that

$$
\begin{aligned}
& \int_{\Omega_{0}} \nabla \varphi_{0} \cdot \nabla\left(\varphi_{0}-u_{0}^{+}\right) d x+\sum_{k=2}^{m} a_{k}\left|B_{k}\right| \int_{D_{k}} \partial_{x_{3}} \varphi_{k} \partial_{x_{3}}\left(\varphi_{k}-u_{0}^{(k)}\right) d x \\
& \quad+\int_{\Omega_{0}} \mu_{0}\left(\varphi_{0}\right)\left(\varphi_{0}-u_{0}^{+}\right) d x+\sum_{k=2}^{m}\left|B_{k}\right| \int_{D_{k}} \mu_{k}\left(\varphi_{k}\right)\left(\varphi_{k}-u_{0}^{(k)}\right) d x \\
& \quad+\sum_{k=2}^{m} \delta_{\alpha_{k}, 1} l_{k} \int_{D_{k}} h_{k}\left(\varphi_{k}\right)\left(\varphi_{k}-u_{0}^{(k)}\right) d x \\
& \geq \int_{\Omega_{0}} f\left(\varphi_{0}-u_{0}^{+}\right) d x
\end{aligned}
$$

for an arbitrary multi-valued function $\varphi \in C_{\mathcal{G}, 0}^{\infty}\left(\Omega_{0}, D_{1}, \ldots, D_{m} ; Q\right)$. Here we have essentially used $h_{1}(0)=0$ and (4.5).

Now let us take any $k_{0} \in\{2, \ldots, m\}$. Due to (4.6) and (4.7) we can consider (4.9) with the following multi-valued function:

$$
\boldsymbol{\varphi}_{k_{0}}(x)= \begin{cases}u_{0}^{+}(x), & x \in \Omega_{0}, \\ 0, & x \in G_{\varepsilon}(1), \\ \varphi_{k_{0}}(x), & x \in G_{\varepsilon}\left(k_{0}\right), \\ u_{0}^{(k)}(x), & x \in G_{\varepsilon}(k), k \in\{2, \ldots, m\} \backslash\left\{k_{0}\right\},\end{cases}
$$

where $\varphi_{k_{0}}$ is arbitrary function from $\mathfrak{K}_{k_{0}}$. As a result, we have

$$
\begin{aligned}
& a_{k_{0}}\left|B_{k_{0}}\right| \int_{D_{k_{0}}} \partial_{x_{3}} \varphi_{k_{0}} \partial_{x_{3}}\left(\varphi_{k_{0}}-u_{0}^{\left(k_{0}\right)}\right) d x+\left|B_{k_{0}}\right| \int_{D_{k_{0}}} \mu_{k_{0}}\left(\varphi_{k_{0}}\right)\left(\varphi_{k_{0}}-u_{0}^{\left(k_{0}\right)}\right) d x \\
& \quad+\delta_{\alpha_{k_{0}}, 1} l_{k_{0}} \int_{D_{k_{0}}} h_{k_{0}}\left(\varphi_{k_{0}}\right)\left(\varphi_{k_{0}}-u_{0}^{\left(k_{0}\right)}\right) d x \\
& \geq 0, \quad \forall \varphi_{k_{0}} \in \mathfrak{K}_{k_{0}} .
\end{aligned}
$$

This variational inequality means that $u_{0}^{\left(k_{0}\right)}$ is the unique solution to the problem (4.3) (see Definition 4.2). Owing to the uniqueness of this solution, the above argumentations are true for any subsequence of $\{\varepsilon\}$ chosen at the beginning of the proof. Thus the limits in (4.1) hold. 


\section{Conclusion}

1. In the Signorini boundary conditions there are two alternative sets of boundary conditions the solution must satisfy and it is not a priori known what of the two sets is satisfied for each point. This type of boundary conditions are most suitable for simulation of different processes in domains with complex structure of the boundary. For our problem (1.2)-(1.3) this is an inhomogeneous Dirichlet condition or a nonlinear Robin condition:

$$
u_{\varepsilon}=g_{k} \quad \text { or } \quad a_{k} \partial_{v} u_{\varepsilon}+\varepsilon^{\alpha_{k}} h_{k}\left(u_{\varepsilon}\right)=0
$$

on the lateral surfaces $S_{\varepsilon}(k)$ of the thin cylinders $G_{\varepsilon}(k)(k=1, \ldots, m)$.

This Robin condition describes the motion of a flux of a quantity having different features that are described by the function $h_{k}$ and by the presence of special intensity factor $\varepsilon^{\alpha_{k}}$. At first glance it may seem that there is no difference between the Robin condition and the homogeneous Neumann condition, since the term $h_{k}\left(u_{\varepsilon}\right)$ is multiplied by $\varepsilon^{\alpha_{k}}$. However, as we can see from Theorems 3.1 and 4.1, this is true only if $\alpha_{k}>1$. If $\alpha_{k}=1$, then the new blow-up term $l_{k} h_{k}\left(u_{0}^{(k)}\right)$ appears in the variational relations in $D_{k}$ (see (3.4) and (4.3)). Just the appearance of this term in the homogenized relations provides mathematical justification of the chemical activity of nanostructural materials (see [8] for more detail).

If physical properties of the thin cylinders from the different classes are almost similar $\left(\alpha_{k} \geq 1, k=1, \ldots, m\right)$, then the global flow described by the multi-valued function $\mathbf{u}_{0}$ (see (3.3)) behaves as a many-phase system in the region which is filled up by the thin cylinder from each class in the limit passage as the parameter $\varepsilon \rightarrow 0$. An analogous effect was observed in the asymptotic behavior of solutions of various boundary-value problems in thick multi-level junctions of different types [12-15].

If some $\alpha_{k_{0}}<1$, then the interaction between the lateral surfaces $S_{\varepsilon}\left(k_{0}\right)$ of the thin cylinders $G_{\varepsilon}\left(k_{0}\right)$ and the outside medium is very intensive and plays a dominant role in the asymptotic behavior of the problem (1.2)-(1.3). Note that this interaction may not be too strong locally if $0 \leq \alpha_{k_{0}}<1$, but it produces this effect due to the total area of the surfaces $S_{\varepsilon}\left(k_{0}\right)$. As a result, the solution $u_{\varepsilon}$ tends to zero in the thin cylinders $G_{\varepsilon}\left(k_{0}\right)$. Since the cylinders $G_{\varepsilon}\left(k_{0}\right)$ are $\varepsilon$-periodically alternated with cylinders from the other classes, the problem (1.2)-(1.3) splits, in passing to the limit as $\varepsilon \rightarrow 0$, into the boundary-value problem (4.2) and the spatial variational inequalities (4.3).

Successful applications of thick-junction constructions have stimulated active study of BVPs in thick junctions with strongly contrasting physical properties (see [16-18]).

2. We can obtain similar results if the functions $\left\{\mu_{k}\right\}_{k=1}^{m}$ and $\left\{h_{k}\right\}_{k=1}^{m}$ depend both on $x_{3} \in$ $\left[-d_{k}, 0\right]$ and $s \in \mathbb{R}$. In this case we have to assume that the inequalities (1.4) are satisfied uniformly regarding $x_{3}$, functions $\left\{\mu_{k}\right\}_{k=1}^{m}$ and $\left\{h_{k}\right\}_{k=1}^{m}$ vanish at $x_{3}=0$, and their derivatives with respect to $x_{3}$ are uniformly bounded.

3. Also we think that the solution to the homogenized problem (3.4) can be constructed by using a penalty formulation and successive iteration similarly to the proposal by Wendland in [1], Section 7.

Competing interests

The authors declare that they have no competing interests.

Authors' contributions

All authors contributed equally to the writing of this paper. 


\section{References}

1. Mel'nyk, TA, Nakvasiuk, IA, Wendland, WL: Homogenization of the Signorini boundary-value problem in a thick junction and boundary integral equations for the homogenized problem. Math. Methods Appl. Sci. 34(7), 758-775 (2011)

2. Feng, ZC: Handbook of Zinc Oxide and Related Material. Volume Two: Devices and Nano-Engineering. CRC Press, Boca Raton (2013)

3. Lenczner, M: Multiscale model for atomic force microscope array mechanical behavior. Appl. Phys. Lett. 90, 091908 (2007)

4. Lyshevshi, SL: Mems and Nems: Systems, Devices, and Structures. CRC Press, Boca Raton (2002)

5. Anish, KM, Soyoun, J, Taeksoo, J: Protein biosensors based on polymer nanowires, carbon nanotubes and zinc oxide nanorods. Sensors 11, 5087-5111 (2011). doi:10.3390/s110505087

6. Kinderlehrer, D, Stampaccia, G: An Introduction to Variational Inequalities and Their Applications. Academic Press, New York (1980)

7. Lions, JL: Quelques méthodes de résolution des problèmes aux limites non linéires. Dunod, Paris (1969)

8. Mel'nyk, TA: Homogenization of a boundary-value problem with a nonlinear boundary condition in a thick junction of type 3:2:1. Math. Models Methods Appl. Sci. 31, 1005-1027 (2008)

9. Cioranescu, D, Saint Jean Paulin, J: Homogenization in open sets with holes. J. Math. Anal. Appl. 71, 590-607 (1979)

10. Conca, C, Diaz, J, Linan, A, Timofte, C: Homogenization in chemical reactive flows. Electron. J. Differ. Equ. 2004, 40 (2004)

11. Pao, CV: Nonlinear Parabolic and Elliptic Equations. Plenum Press, New York (1992)

12. De Maio, U, Durante, T, Mel'nyk, TA: Asymptotic approximation for the solution to the Robin problem in a thick multi-level junction. Math. Models Methods Appl. Sci. 15(12), 1897-1921 (2005)

13. Durante, T, Mel'nyk, TA: Homogenization of quasilinear optimal control problems involving a thick multilevel junction of type $3: 2: 1$. ESAIM Control Optim. Calc. Var. 18(2), 583-610 (2012)

14. Mel'nyk, TA: Asymptotic approximation for the solution to a semi-linear parabolic problem in a thick junction with the branched structure. J. Math. Anal. Appl. 424, 1237-1260 (2015)

15. Mel'nyk, TA, Sadovyj, DI: Homogenization of a quasilinear parabolic boundary problem with different alternating nonlinear boundary Fouirer boundary conditions in a two-level thick junction of the type 3:2:2. Ukr. Math. J. 63(12) 1855-1882 (2012)

16. Mel'nyk, TA, Nazarov, SA: Asymptotic analysis of the Neumann problem on the junction of a body and thin heavy rods. St. Petersburg Math. J. 12(2), 317-351 (2001)

17. Mel'nyk, TA, Chechkin, GA: Eigenvibrations of thick cascade junctions with "super heavy" concentrated masses. Izv. RAN. Ser. Mat. 79(3), 41-86 (2015)

18. Gaudiello, A, Sili, A: Homogenization of highly oscilating boundaries with strongly contrasting diffusivity. SIAM J. Math. Anal. 47(3), 1671-1692(2015)

\section{Submit your manuscript to a SpringerOpen ${ }^{\circ}$ journal and benefit from:}

- Convenient online submission

- Rigorous peer review

- Immediate publication on acceptance

Open access: articles freely available online

High visibility within the field

- Retaining the copyright to your article 